\title{
Symmetry and Antisymmetry of the CMB Anisotropy Pattern
}

\author{
Jaiseung Kim, Pavel Naselsky, and Martin Hansen \\ Discovery Center, Niels Bohr Institute, Blegdamsvej 17, 2100 Copenhagen, Denmark \\ Correspondence should be addressed to Jaiseung Kim, jkim@nbi.dk
}

Received 3 February 2012; Accepted 2 June 2012

Academic Editor: Alberto J. Castro-Tirado

Copyright () 2012 Jaiseung Kim et al. This is an open access article distributed under the Creative Commons Attribution License, which permits unrestricted use, distribution, and reproduction in any medium, provided the original work is properly cited.

\begin{abstract}
Given an arbitrary function, we may construct symmetric and antisymmetric functions under a certain operation. Since statistical isotropy and homogeneity of our Universe has been a fundamental assumption of modern cosmology, we do not expect any particular symmetry or antisymmetry in our Universe. Besides fundamental properties of our Universe, we may also figure our contamination and improve the quality of the $\mathrm{CMB}$ data products, by matching the unusual symmetries and antisymmetries of the $\mathrm{CMB}$ data with known contaminantions. If we let the operation to be a coordinate inversion, the symmetric and antisymmetric functions have even and odd-parity respectively. The investigation on the parity of the recent CMB data shows a large-scale oddparity preference, which is very unlikely in the statistical isotropic and homogeneous Universe. We investigated the association of the WMAP systematics with the anomaly, but did not find a definite non-cosmological cause. Besides the parity anomaly, there is anomalous lack of large-scale correlation in CMB data. We show that the odd-parity preference at low multipoles is, in fact, phenomenologically identical with the lack of large-angle correlation.
\end{abstract}

\section{Introduction}

In an inflationary paradigm, the $\mathrm{CMB}$ anisotropy pattern is expected to follow a random Gaussian distribution with the statistical isotropy and homogeneity, due to the nature of the quantum fluctuation during the cosmic inflation [1-5]. Passing through the period of the inflation and cosmological evolution from very early stages till the present day, the quantum fluctuations turn into classical fluctuation, in which all information about the beginning of the inflation, the ionization history of the cosmic plasma, and the formation of the large-scale structure have been well preserved. Therefore, the observation of the CMB anisotropy allows us to investigate the extreme states of matter and radiation well beyond the limit, obtainable by modern particle accelerators and shed light on the problem of "darkness" of the Universe, in which the present mass density mainly consists of the cold dark matter and the dark energy.

For the past years, there have been great successes in measurement of $\mathrm{CMB}$ anisotropy by ground and satellite observations [6-16]. Since release of the data from the orbital observations [17-19], the issue of statistical anisotropy and non-Gaussianity has been given very significant attention. Several hints of statistical anisotropy and non-Gaussianity have been reported [20-43]. In particular, many of the reported anomalies are associated with low multipoles $(2 \leq$ $l \leq 30)$ of the CMB, including the low amplitude of the quadrupole [44] and the striking alignment between the quadrupole and octupole, dubbed the "axis of evil" [27, 28, 44 ], and some other features of the CMB map [42] and the power spectrum [37, 38]. These anomalies could be given two possible explanations. The first one is that statistical homogeneity and isotropy of the primordial fluctuation in general is obeyed, but we are living in a Universe, which is not typical of the ensemble Universe. The second explanation is that, at least for some range of multipoles, the properties of primordial fluctuations are in disagreement with the isotropic Gaussian Universe.

The CMB anisotropy at low multipoles are associated with scales far beyond any existing astrophysical survey, and therefore $\mathrm{CMB}$ anomalies at low multipoles may hint new physics at unexplored large scales, including nontrivial topology of the Universe, broken scale invariance at large scales. On the other hand, these anomalies are simply due to noncosmological contamination such as unaccounted astrophysical emission (e.g., the Kuiper Belt objects) and unknown systematic effects.

Recently, it was shown that some of the anomalies can be explained in terms of symmetries and antisymmetries of 
the CMB sky [37, 38, 41]. For instance, the CMB anisotropy pattern may be considered as the sum of symmetric and antisymmetric functions under the coordinate inversion. Equivalently, the forementioned symmetric and antisymmetric functions possess even and odd parity, respectively. Given the Gaussian Universe, we do not expect the CMB anisotropy pattern to show a particular parity preference. However, the angular power spectrum of WMAP data shows anomalous odd-parity preference at low multipoles [37$40,45]$. In this work, we are going to discuss the odd-parity preference of the WMAP data and present our investigation on its origins. In order to understand the nature of the odd-parity preference, we have additionally investigated the phase of even and odd multipole data, respectively, and found they show features distinct from each other. The parity anomaly is explicitly associated with the angular power spectrum, which is heavily used for cosmological model fitting. Having noted this, we have also fitted a cosmological model, respectively, to even and odd multipole data set and found significant tension [40]. These parametric tensions indicate either unaccounted contamination or insufficiency of the assumed model.

One of most important elements in the study of nonGaussianity is to identify the anomalies of the common origin, whether it is cosmological or systematics. In particular, there have been reports on the lack of large-angle correlation, since the COBE-DMR data [30,46-50]. Recently, we have shown that the lack of large-angle correlation is phenomenologically identical with the odd-parity anomaly of the $\mathrm{CMB}$ power spectrum. Besides them, we may understand the low quadrupole power as a part of odd-parity preference at low multipoles [37, 38, 44]. Even though it still leaves the fundamental question on its origin unanswered, the association between seemingly distinct anomalies will help the investigation on the underlying origin.

The outline of the paper is the following. In Section 2, we discuss the anomalous odd-parity preference of the WMAP data. In Section 3, we investigate the phase of the even and odd multipole data, respectively, and discuss its result. In Section 4, we investigate the octupole component of CMB anisotropy and discuss some anomalous feature. In Section 5, we show there is a significant parametric tension between the cosmological models, when fitted to the even or odd low multipole data, respectively. In Sections 6 and 7 , we discuss the lack of correlation of WMAP data at large and small angles and show the odd-parity preference at low multipoles is phenomenologically identical with the lack of the large-angle correlation. Finally, in Section 8, we discuss the findings and draw our conclusions.

\section{Parity Asymmetry of the WMAP Data}

The CMB temperature anisotropy over a whole-sky is conveniently decomposed in terms of spherical harmonics $Y_{l m}(\theta, \phi)$ as follows:

$$
T(\widehat{\mathbf{n}})=\sum_{l m} a_{l m} Y_{l m}(\widehat{\mathbf{n}})
$$

where $a_{l m}$ is a decomposition coefficient and $\hat{\mathbf{n}}$ is a sky direction. Decomposition coefficients are related to primordial perturbation as follows:

$$
a_{l m}=4 \pi(-\imath)^{l} \int \frac{d^{3} \mathbf{k}}{(2 \pi)^{3}} \Phi(\mathbf{k}) g_{l}(k) Y_{l m}^{*}(\hat{\mathbf{k}})
$$

where $\Phi(\mathbf{k})$ is primordial perturbation in Fourier space and $g_{l}(k)$ is a radiation transfer function. For a Gaussian model for primordial perturbation, decomposition coefficients satisfy the following statistical properties:

$$
\begin{gathered}
\left\langle a_{l m}\right\rangle=0, \\
\left\langle a_{l m}^{*} a_{l^{\prime} m^{\prime}}\right\rangle=C_{l} \delta_{l l^{\prime}} \delta_{m m^{\prime}},
\end{gathered}
$$

where $\langle\cdots\rangle$ denotes the average over the ensemble of universes. Given a standard cosmological model, Sach-Wolf plateau is expected at low multipoles [2]: $l(l+1) C_{l} \sim$ const.

From the CMB anisotropy, we may construct a symmetric and antisymmetric function under the coordinate inversion $\mathbf{n} \rightarrow-\mathbf{n}$ :

$$
\begin{aligned}
T^{+}(\widehat{\mathbf{n}}) & =\frac{T(\hat{\mathbf{n}})+T(-\widehat{\mathbf{n}})}{2}, \\
T^{-}(\widehat{\mathbf{n}}) & =\frac{T(\hat{\mathbf{n}})-T(-\widehat{\mathbf{n}})}{2} .
\end{aligned}
$$

In other words, $T^{+}(\widehat{\mathbf{n}})$ and $T^{-}(\widehat{\mathbf{n}})$ have even and odd parity. Taking into account the parity property of spherical harmonics $Y_{l m}(\widehat{\mathbf{n}})=(-1)^{l} Y_{l m}(-\widehat{\mathbf{n}})[51]$, we may easily show

$$
\begin{aligned}
& T^{+}(\hat{\mathbf{n}})=\sum_{l m} a_{l m} Y_{l m}(\widehat{\mathbf{n}}) \cos ^{2}\left(\frac{l \pi}{2}\right), \\
& T^{-}(\hat{\mathbf{n}})=\sum_{l m} a_{l m} Y_{l m}(\widehat{\mathbf{n}}) \sin ^{2}\left(\frac{l \pi}{2}\right),
\end{aligned}
$$

where $n$ is an integer. Therefore, significant power asymmetry between even and odd multipoles may be interpreted as a preference for a particular parity of the anisotropy pattern. Hereafter, we will denote a preference for particular parity by "parity asymmetry." In Figure 1, we show the WMAP 7year, 5-year,and 3-year data and the WMAP concordance model $[6,9,52-54]$. From Figure 1, we may see that the power spectrum of WMAP data at even multipoles tend to be lower than those at neighboring odd multipoles. In Figure 2, we show $(-1)^{l} l(l+1) / 2 \pi\left(C_{l}^{\mathrm{WMAP}}-C_{l}^{\Lambda \mathrm{CDM}}\right)$ for low multipoles. Since we expect random scattering of data points around a theoretical model, we expect the distribution of dots in Figure 2 to be scattered around the both side of zero. However, there are only 5 points of positive values among 22 points in the case of WMAP7 or WMAP5 data. Therefore, we may see that there is the tendency of power deficit (excess) at even (odd) multipoles, compared with the $\Lambda$ CDM model. 


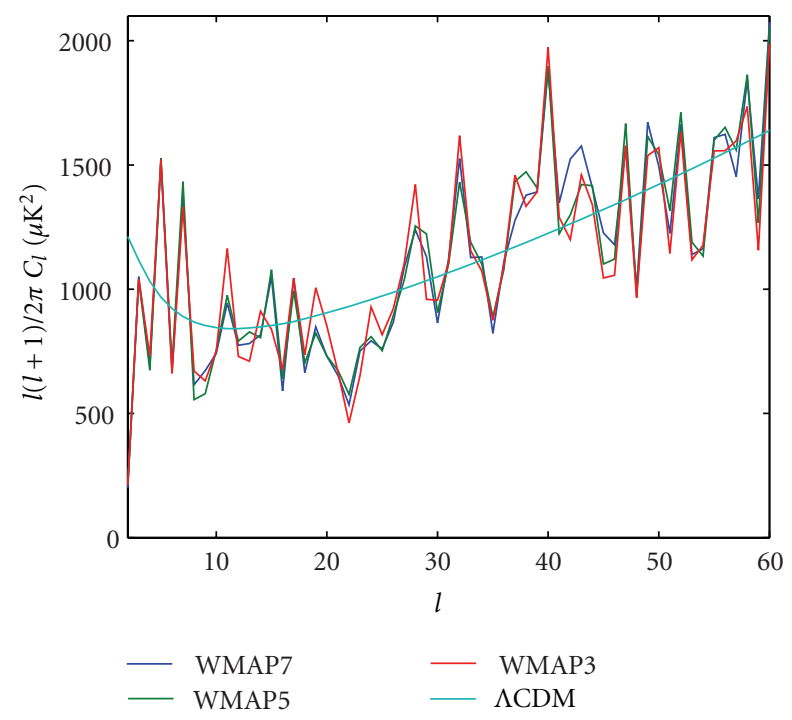

FIgURe 1: CMB power spectrum: WMAP 7 year data (blue), WMAP 5 year data (green) and WMAP 3 year data (red), $\Lambda$ CDM model (cyan).

Taking into account $l(l+1) C_{l} \sim$ const, we may consider the following quantities:

$$
\begin{aligned}
& P^{+}=\sum_{l=2}^{l_{\max }} \cos ^{2}\left(\frac{l \pi}{2}\right) \frac{l(l+1)}{2 \pi} C_{l}, \\
& P^{-}=\sum_{l=2}^{l_{\max }} \sin ^{2}\left(\frac{l \pi}{2}\right) \frac{l(l+1)}{2 \pi} C_{l},
\end{aligned}
$$

where $P^{+}$and $P^{-}$are the sum of $l(l+1) / 2 \pi C_{l}$ for even and odd multipoles, respectively. Therefore, the ratio $P^{+} / P^{-}$is associated with the degree of the parity asymmetry, where the lower value of $P^{+} / P^{-}$indicates odd-parity preference, and vice versa.

In Figure 3, we show the $P^{+} / P^{-}$of WMAP data, and a $\Lambda \mathrm{CDM}$ model for various $l_{\max }$. As shown in Figure $3, P^{+} / P^{-}$ of WMAP data are far below theoretical values. Though the discrepancy is largest at lowest $l_{\max }$, its statistical significance is not necessarily high for low $l$, due to associated statistical fluctuation. In order to make a rigorous assessment on its statistical significance at low $l$, we compared $P^{+} / P^{-}$of WMAP data with that of simulation. We have produced $10^{4}$ simulated CMB maps of HEALPix Nside $=8$ and Nside $=$ 512, respectively, via map synthesis with $a_{l m}$ randomly drawn from Gaussian $\Lambda$ CDM model. We have degraded the WMAP processing mask $(N$ side $=16)$ to $N$ side $=8$, and set pixels to zero, if any of their daughter pixels is zero. After applying the mask, we have estimated power spectrum $2 \leq l \leq 23$ from simulated cut-sky maps $(N$ side $=8)$ by a pixel-based maximum likelihood method $[6,55,56]$. At the same time, we have applied the WMAP team's KQ85 mask to the simulated maps $(N$ side $=512)$ and estimated power spectrum $2 \leq l \leq 1024$ by pseudo $C_{l}$ method $[57,58]$. In the simulation, we have neglected instrument noise, since the signal-to-noise ratio of the WMAP data is

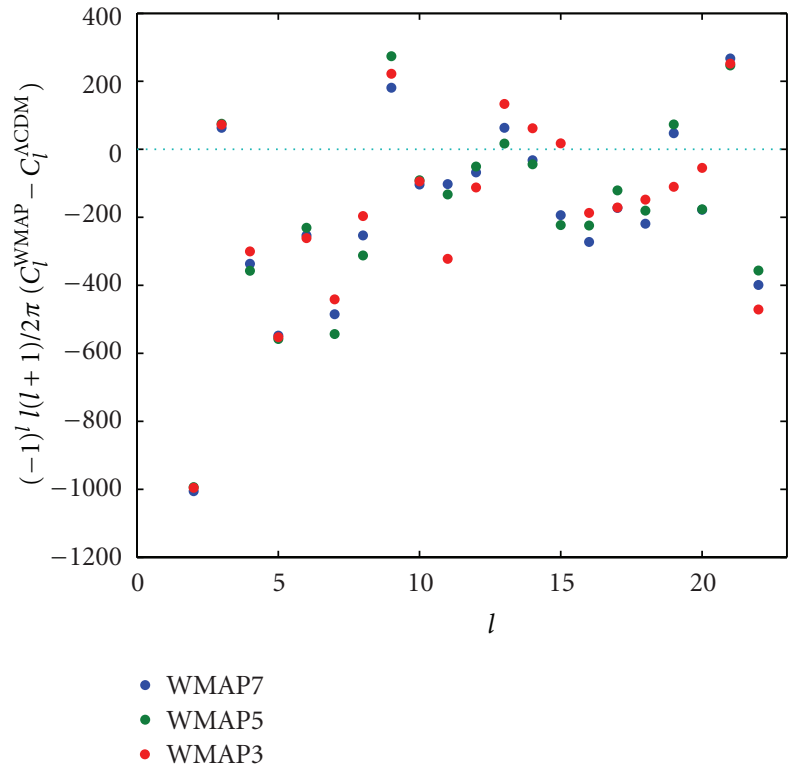

Figure $2:(-1)^{l} \times$ difference between WMAP power spectrum data and $\Lambda$ CDM model.

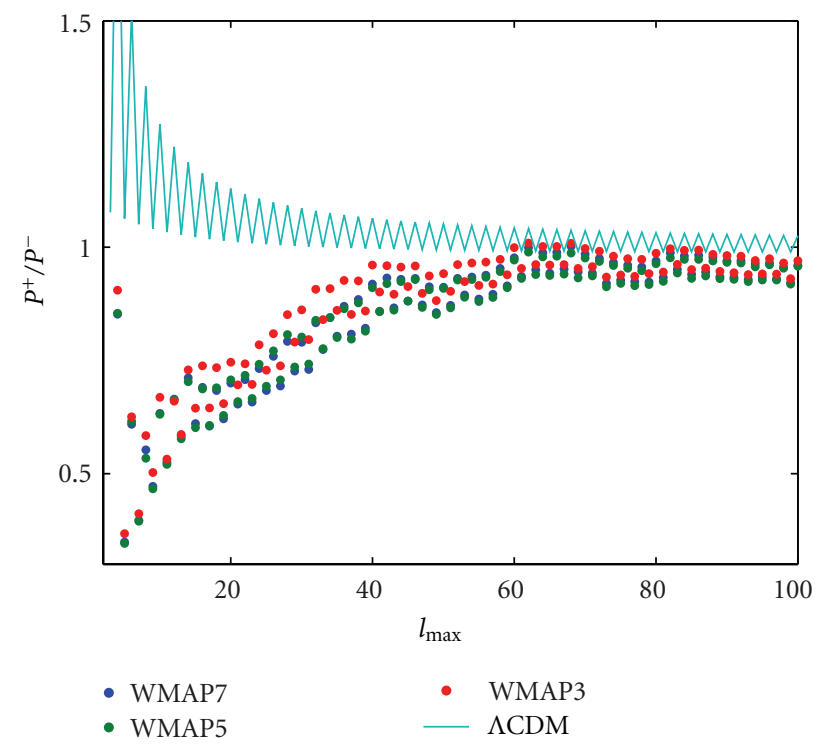

Figure $3: P^{+} / P^{-}$of WMAP data and $\Lambda \mathrm{CDM}$.

quite high at multipoles of interest (i.e., $l \leq 100)[6,7]$. Using the low $l$ estimation by pixel-maximum likelihood method and high $l$ estimation by pseudo $C_{l}$ method, we have computed $P^{+} / P^{-}$, respectively, for various multipole ranges $2 \leq l \leq l_{\max }$ and compared $P^{+} / P^{-}$of the WMAP data with simulation. In Figure 4, we show $P$ value of WMAP7, WMAP5 and WMAP3, respectively, for various $l_{\max }$, where $P$-value denotes fractions of simulations as low as $P^{+} / P^{-}$of the WMAP data. As shown in Figure 4 , the parity asymmetry of WMAP7 data at multipoles $(2 \leq l \leq 22)$ is most anomalous, where $P$-value is 0.0031 . As shown in Figure 4, the statistical significance of the parity asymmetry (i.e., low $P$-value) is getting higher, when we increase $l_{\max }$ up to 22 . 


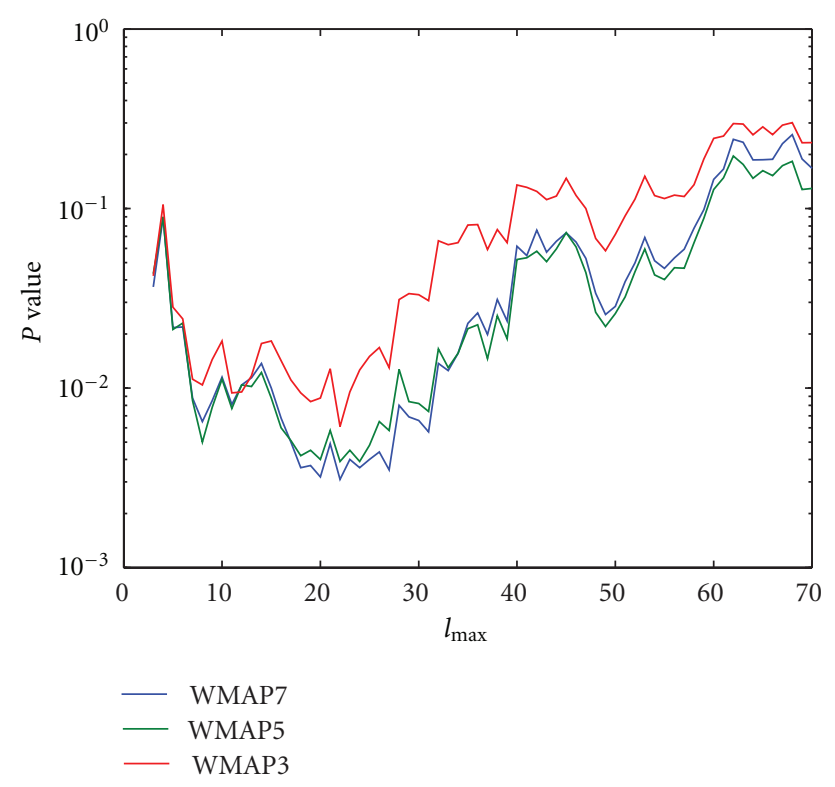

Figure 4: Probability of getting $P^{+} / P^{-}$as low as WMAP data for multipole range $2 \leq l \leq l_{\max }$.

TABLE 1: The parity asymmetry of WMAP data $(2 \leq l \leq 22)$.

\begin{tabular}{lll}
\hline Data & $P^{+} / P^{-}$ & $P$ value \\
\hline WMAP7 & 0.7076 & 0.0031 \\
WMAP5 & 0.7174 & 0.0039 \\
WMAP3 & 0.7426 & 0.0061 \\
\hline
\end{tabular}

Therefore, we may not attribute the odd parity preference simply to the low quadrupole power and find it rather likely that the low quadrupole power is not an isolated anomaly, but shares an origin with the odd parity preference.

In Table 1 , we summarize $P^{+} / P^{-}$and $P$ values of WMAP7, WMAP5, and WMAP3 for $l_{\max }=22$. As shown in Figure 4 and Table 1, the odd-parity preference of WMAP7 is most anomalous, while WMAP7 data are believed to have more accurate calibration and less foreground contamination than earlier releases $[6-9,59]$. In Figure 5, we show cumulative distribution of $P^{+} / P^{-}$for $10^{4}$ simulated maps. The values corresponding to $P^{+} / P^{-}$of WMAP data are marked as dots.

In the absence of strong theoretical grounds for the parity asymmetry $(2 \leq l \leq 22)$, we have to take into account our posteriori choice on $l_{\max }$, which might have enhanced the statistical significance. However, as shown in Figure 4, the odd-parity preference exists for various values of $l_{\max }$. Therefore, the statistical enhancement by our posterior choice on $l_{\max }$ is not significant.

2.1. Cosmological or Noncosmological? In the WMAP data, there are noncosmological contamination such as asymmetric beams, instrument noise, foreground, and cut-sky effect, which might be responsible for the discussed anomaly. First of all, there is contamination from galactic and extragalactic foregrounds. In order to reduce foreground contamination,

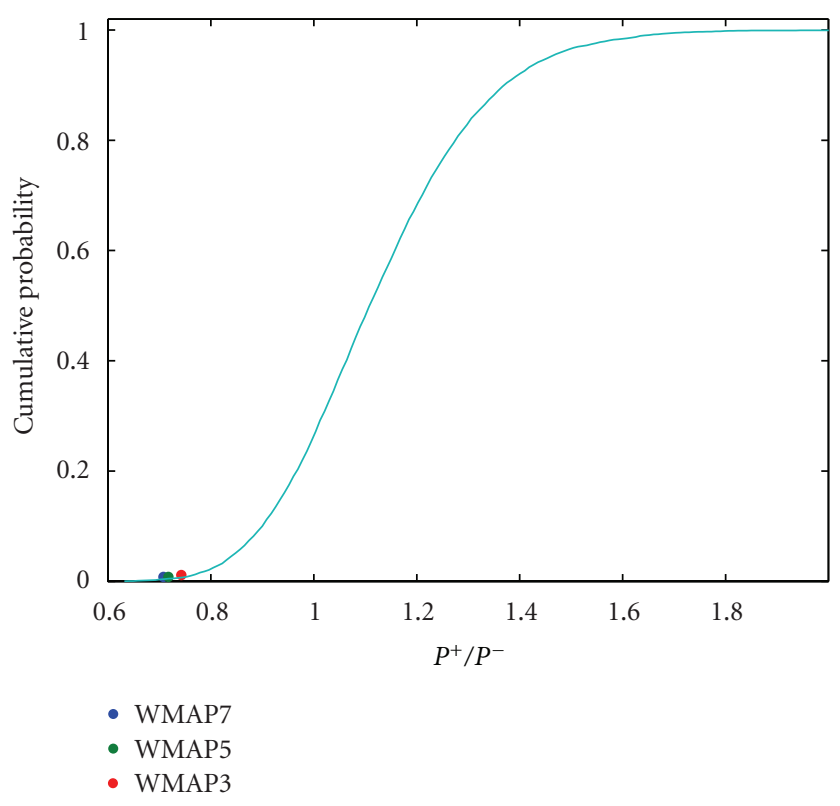

Figure 5: Parity asymmetry at multipoles $(2 \leq l \leq 22)$ : cumulative distribution of $P^{+} / P^{-}$for $10^{4}$ simulated maps (cyan), $P^{+} / P^{-}$of WMAP7 (blue), WMAP5 (green), and WMAP3 (red).

the WMAP team have subtracted diffuse foregrounds by template-fitting and masked the regions that cannot be cleaned reliably. For foreground templates (dust, free-free emission and synchrotron), the WMAP team used dust emission "Model 8," $\mathrm{H} \alpha$ map, and the difference between $\mathrm{K}$ and Ka band maps [8, 60-63]. In Figure 6, we show the power spectrum of templates. As shown in Figure 6, templates show strong even parity preference, which is opposite to that of the WMAP power spectrum data. Therefore, one might wrongly attribute the odd-parity preference of WMAP data to oversubtraction by templates. Consider spherical harmonic coefficients of a foreground-reduced map:

$$
a_{l m}^{\mathrm{obs}}=a_{l m}^{\mathrm{cmb}}+a_{l \mathrm{~m}}^{\mathrm{fg}}-b a_{l m}^{\mathrm{tpl}}
$$

where $a_{l m}^{\mathrm{obs}}, a_{l m}^{\mathrm{fg}}$, and $b a_{l m}^{\mathrm{tpl}}$ correspond to a foregroundcleaned map, a foreground, and a template with a fitting coefficient $b$. For simplicity, we consider only a single foreground component, but the conclusion is equally valid for multicomponent foregrounds. Since there is no correlation between foregrounds and $\mathrm{CMB}$, the observed power spectrum is given by

$$
C_{l}^{\mathrm{obs}} \approx C_{l}^{\mathrm{cmb}}+\left\langle\left|a_{l m}^{\mathrm{fg}}-b a_{l m}^{\mathrm{tpl}}\right|^{2}\right\rangle .
$$

As shown (8), the parity preference should follow that of templates (i.e., even parity preference), because of the second term, provided templates are good tracers of foregrounds (i.e., $a_{l m}^{\mathrm{fg}} / a_{l m}^{\mathrm{tpl}} \approx$ const). Nevertheless, $(8)$ may make a bad approximation for lowest multipoles, because the cross term $\sum_{m} \operatorname{Re}\left[a_{l m}^{\mathrm{cmb}}\left(a_{l m}^{\mathrm{fg}}-b a_{l m}^{\mathrm{tpl}}\right)^{*}\right]$ may not be negligible. Besides that, our argument and the template-fitting method itself 


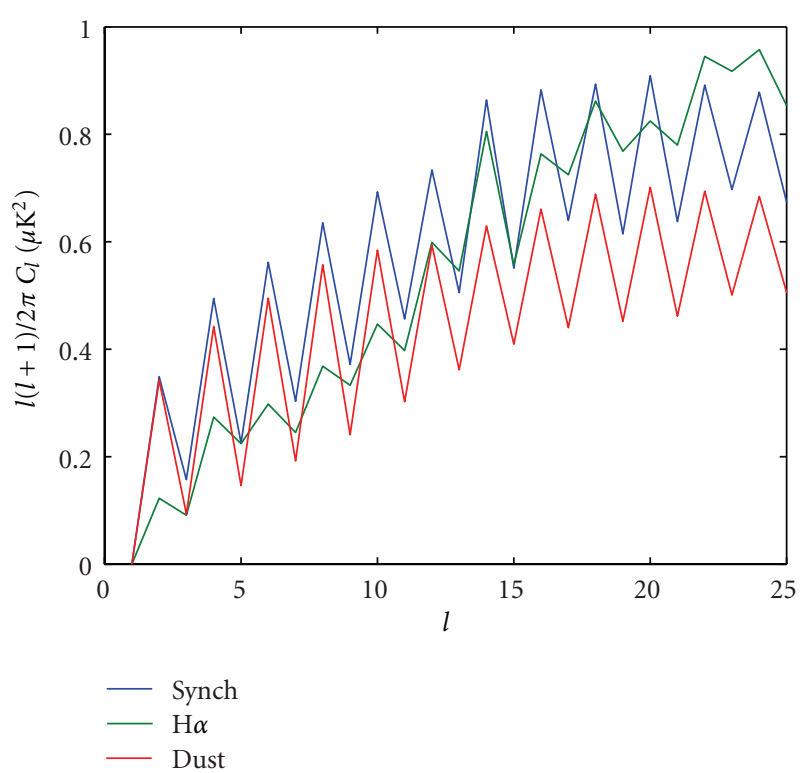

FIGURE 6: The power spectra of the templates (synchrotron, $\mathrm{H} \alpha$, dust): plotted with arbitrary normalization.

fail, if templates are not good tracers of foregrounds. In order to investigate these issues, we have resorted to simulation in combination with WMAP data. Noting the WMAP power spectrum is estimated from foreground-reduced V and W band maps, we have produced simulated maps as follows:

$$
T(\widehat{\mathbf{n}})=T_{\mathrm{cmb}}(\widehat{\mathbf{n}})+\frac{(V(\hat{\mathbf{n}})-W(\widehat{\mathbf{n}}))}{2},
$$

where $V(\widehat{\mathbf{n}})$ and $W(\widehat{\mathbf{n}})$ are foreground-reduced $\mathrm{V}$ and $\mathrm{W}$ band maps of WMAP data. Note that the second term on the right hand side mainly contains residual foregrounds. Just as cut-sky simulation described in Section 2, we have applied a foreground mask to the simulated maps and estimated the power spectrum from cut-sky by a pixel-based maximum likelihood method. In Figure 7, we show $P^{+}$and $P^{-}$values estimated from simulations. For comparison, we have included simulations without residual foregrounds, and dashed lines of a slope corresponding to $P^{+} / P^{-}$of $\Lambda \mathrm{CDM}$ model and WMAP7 data. As shown in Figure 7, the $P^{+} / P^{-}$ of simulations in the presence of residual foregrounds do not show anomalous odd-parity preference of WMAP data. Considering (8) and simulations, we find it difficult to attribute the odd-parity preference to residual foreground. There also exist contamination from unresolved extragalactic point sources [52]. However, point sources follow Poisson distribution with little departure [64] and therefore are unlikely to possess odd-parity preference. Besides that, point sources at WMAP frequencies are subdominant on large angular scales (low $l$ ) $[52,60,64,65]$. Though we have not found association of foregrounds with the anomaly, we do not completely rule out residual foreground, due to our limited knowledge on residual foregrounds.

The WMAP team have masked the region that cannot be reliably cleaned by template fitting and estimated $\mathrm{CMB}$

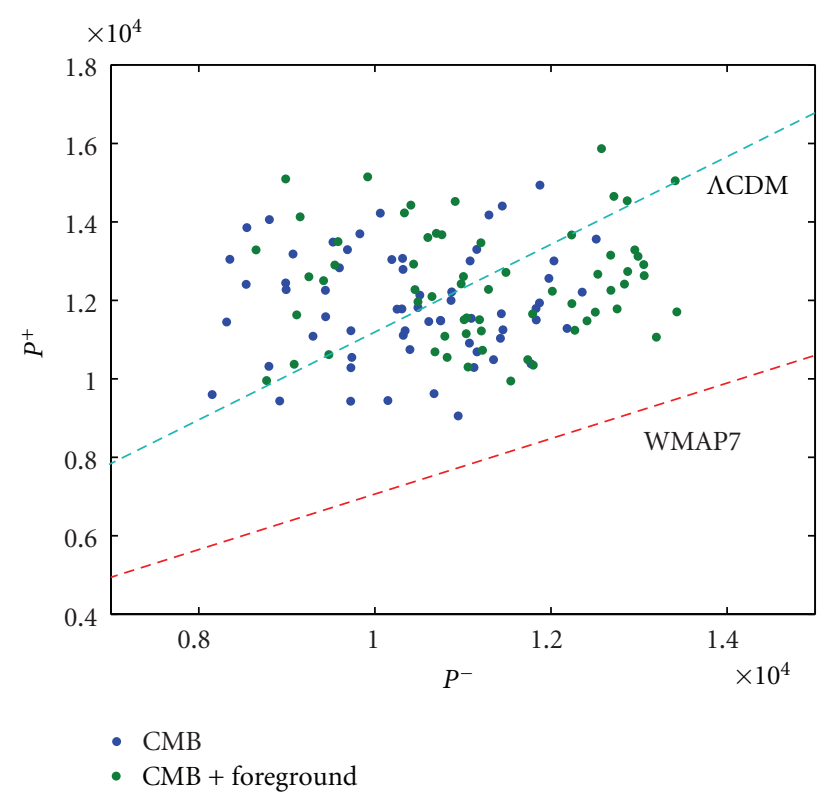

FIGURE 7: The parity asymmetry in the presence of residual foregrounds $(\mathrm{V}-\mathrm{W})$ : dashed lines are plotted with slopes corresponding to $P^{+} / P^{-}$of $P^{+} / P^{-}$of $\Lambda$ CDM (cyan), WMAP7 data (red).

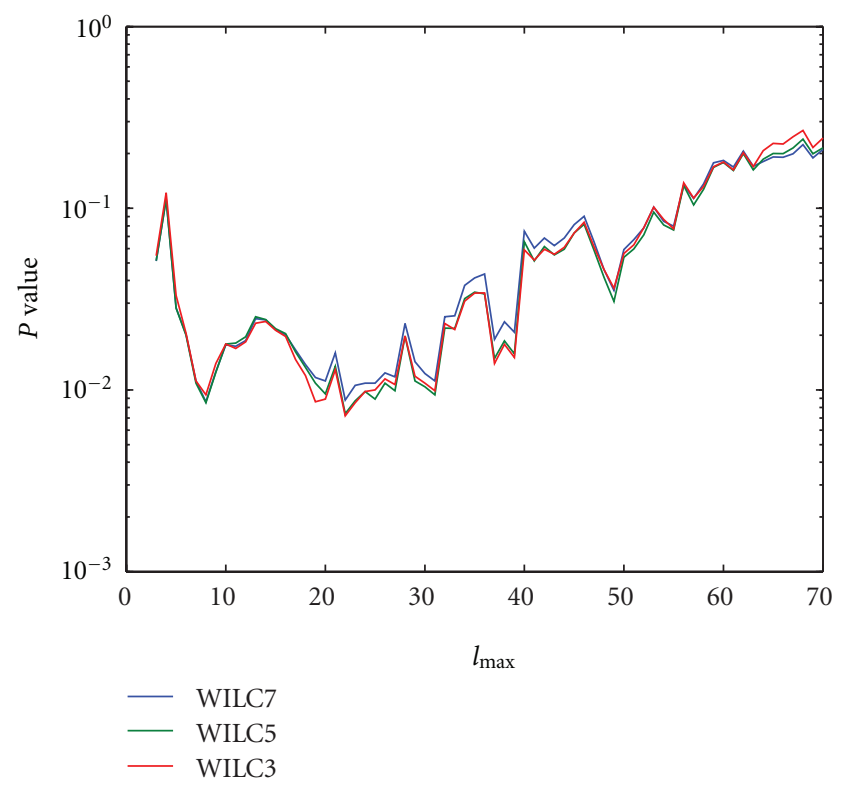

FIgure 8: Probability of getting $P^{+} / P^{-}$as low as the ILC 7-year, 5year, and, 3-year map at multipole range $2 \leq l \leq l_{\max }$.

power spectrum from sky data outside the mask $[6,9,52,60]$. Even though we have properly taken into account the cutsky effect in the $P$ value estimation, we have investigated the WMAP team's Internal Linear Combination (ILC) map, which is expected to provide a reliable estimate of $\mathrm{CMB}$ signal over whole-sky on angular scales larger than $10^{\circ}$ $[52,60,65]$. We have compared $P^{+} / P^{-}$of the ILC maps with whole-sky simulations. In Figure 8, we show $P$-values of the 
TABLE 2: The parity asymmetry of WMAP ILC maps $(2 \leq l \leq 22)$.

\begin{tabular}{llc}
\hline Data & $P^{+} / P^{-}$ & $P$ value \\
\hline ILC7 & 0.7726 & 0.0088 \\
ILC5 & 0.7673 & 0.0074 \\
ILC3 & 0.7662 & 0.0072 \\
\hline
\end{tabular}

ILC maps, respectively, for various $l_{\max }$. As shown in Figure 8, the odd-parity preference of ILC maps is most anomalous for $l_{\max }=22$ as well. In Table 2 , we summarize $P^{+} / P^{-}$and $P$-values for $l_{\max }=22$. As shown in Figure 8 and Table 2, we find anomalous odd-parity preference exits in whole-sky $\mathrm{CMB}$ maps as well. Therefore, we find it difficult to attribute the anomaly to cut-sky effect.

There are instrument noise in the WMAP data. Especially, 1/f noise, when coupled with WMAP scanning pattern, may result in less accurate measurement at certain low multipoles [52, 66, 67]. In order to investigate the association of noise with the anomaly, we have produced noise maps of WMAP7 data by subtracting one Differencing Assembly (D/A) map from another D/A data of the same frequency channel. In Figure 9, we show $P^{+}$and $P^{-}$values of the noise maps. As shown in Figure 9, the noise maps do not show odd-parity preference, but their $P^{+} / P^{-}$ratios are consistent with that of white noise (i.e., $C_{l}=$ const). Besides that, the signal-to-noise ratio of WMAP temperature data is quite high at low multipoles (e.g., $\mathrm{S} / \mathrm{N} \sim 100$ for $l=30)[52,59,67]$. Therefore, we find that instrument noise, including $1 / f$ noise, is unlikely to be the cause of the oddparity preference.

The shape of the WMAP beams is slightly asymmetric $[59,68,69]$, while the WMAP team have assumed symmetric beams in the power spectrum estimation $[6,9,59,68]$. We have investigated the association of beam asymmetry with the anomaly, by using simulated maps provided by [69]. The authors have produced 10 simulated maps for each frequency and Differencing Assembly (D/A) channels, where the detailed shape of the WMAP beams and the WMAP scanning strategy are taken into account [69]. From simulated maps, we have estimated $P^{+}$and $P^{-}$, where we have compensated for beam smoothing purposely by the WMAP team's beam transfer function (i.e., symmetric beams). In Figure 10, we show $P^{+}$and $P^{-}$values of the simulated maps, and the dashed lines of a slope corresponding to $P^{+} / P^{-}$ of $\Lambda \mathrm{CDM}$ and WMAP7 data, respectively. As shown in Figure 10, we do not observe the odd-parity preference of WMAP data in simulated maps. Therefore, we find it hard to attribute the odd-parity preference to asymmetric beams.

Besides contamination discussed so far, there are other sources of contamination such as far sidelobe pickup. In order to investigate these effects, we have resorted to simulation produced by the WMAP team. According to the WMAP team, time-ordered data (TOD) have been simulated with realistic noise, thermal drifts in instrument gains and baselines, smearing of the sky signal due to finite integration time, transmission imbalance, and far-sidelobe beam pickup.

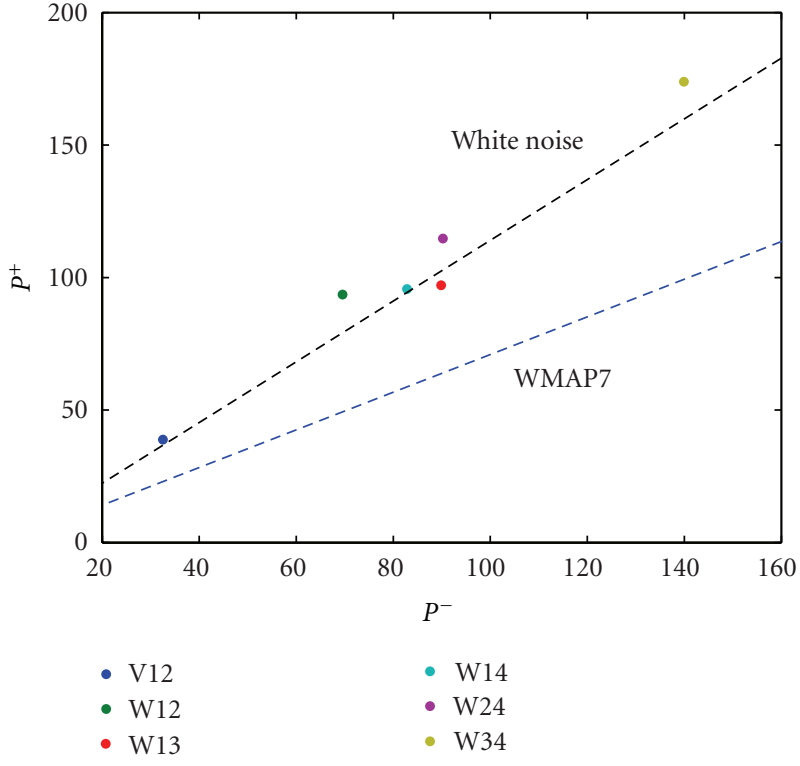

Figure 9: The parity asymmetry of the WMAP noise: the dots denote $\left(P^{+}, P^{-}\right)$of noise maps, and alphanumeric values in the legend denote the frequency band and the pair of D/A channels used. Two dashed lines are plotted with the slope corresponding to $P^{+} / P^{-}$ of white noise and WMAP7 data, respectively.

Using the same data pipeline used for real data, the WMAP team have processed simulated TOD and produced maps for each differencing assembly and each single year observation year. In Figure 11, we show the $P^{+}$and $P^{-}$of the simulated maps, where the power spectrum estimation is made from cut-sky by a pixel-based likelihood method. As shown in Figure 11, all points are far above $P^{+} / P^{-}$of WMAP7 and agree with $\Lambda \mathrm{CDM}$ model. Therefore, we do not find definite association of the parity asymmetry with known systematics effects.

As discussed, we are unable to find a definite noncosmological cause of the anomaly. Therefore, we are going to take the WMAP power spectrum at face values and consider a possible cosmological origin. Topological models including multiconnected Universe and Bianchi VII model have been proposed to explain the cold spot or low quadrupole power [70-72]. However, the topological models do not produce the parity asymmetry, though some of them, indeed, predict low quadrupole power. Trans-Planckian effects and some inflation models predict oscillatory features in primordial power spectrum [4, 73-82]. However, oscillatory or sharp features in primordial power spectrum are smeared out in translation to the CMB power spectrum [20]. Besides, reconstruction of primordial power spectrum and investigation on features show that primordial power spectrum is close to a featureless power-law spectrum $[6,53,54,83-$ 85]. Therefore, we find it difficult to attribute the anomaly to trans-Planckian effect or extended inflation models. We will consider what the odd-parity preference imply on 


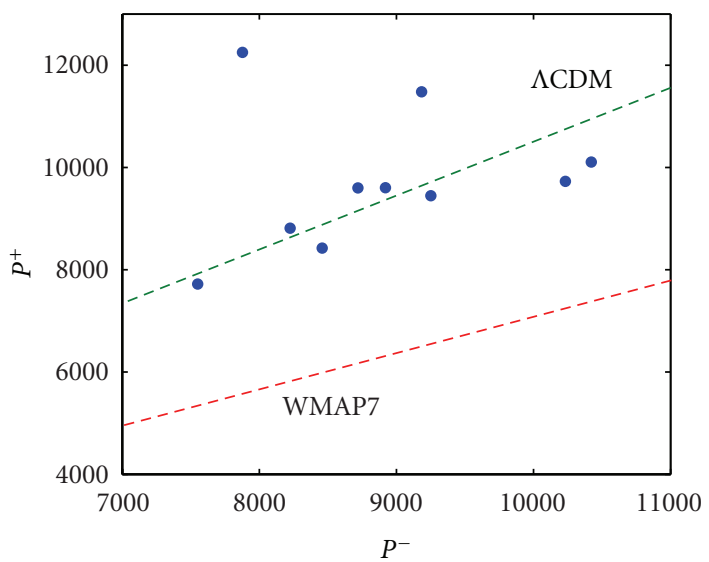

- V1

(a)

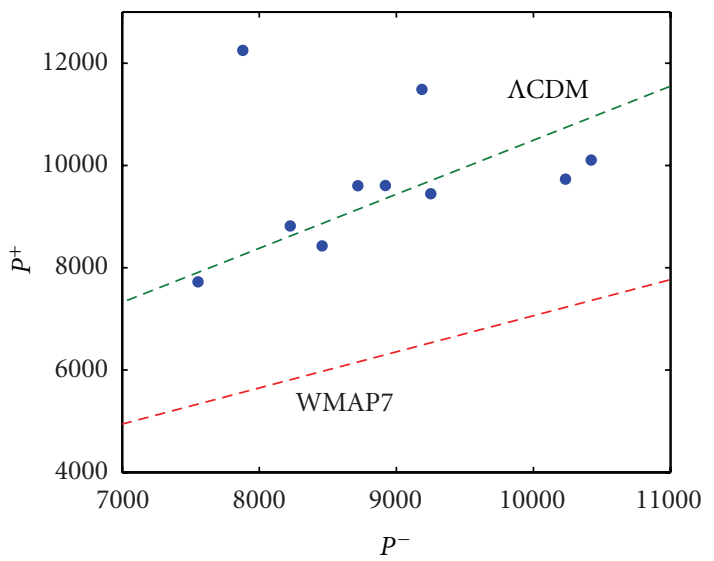

- W1

(c)

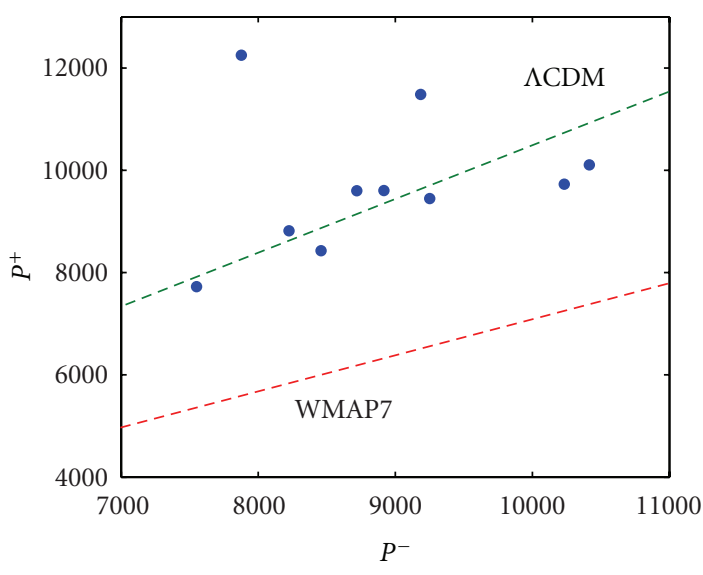

- W3

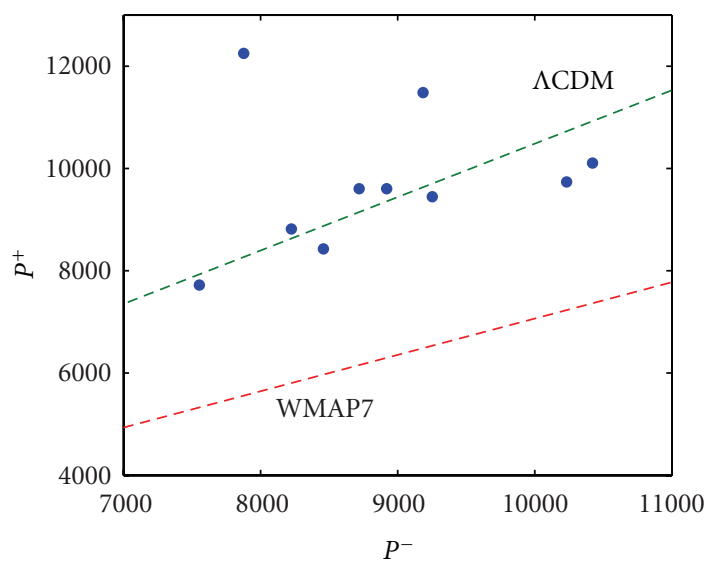

- V2

(b)

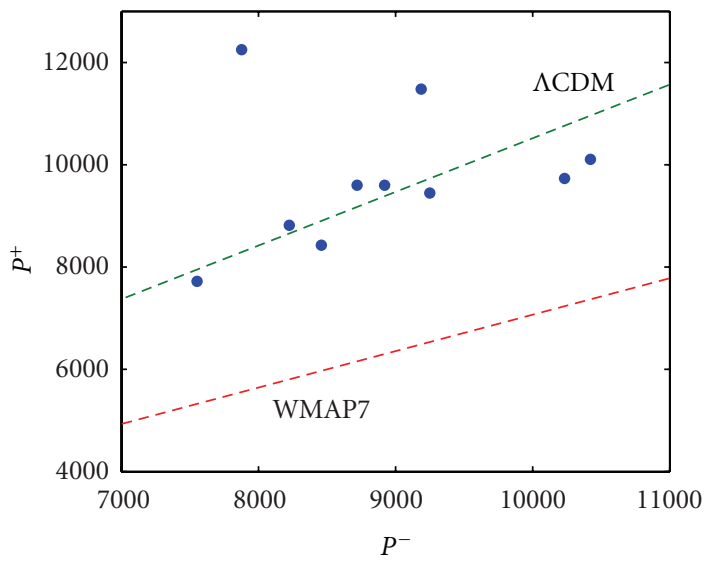

- W2

(d)

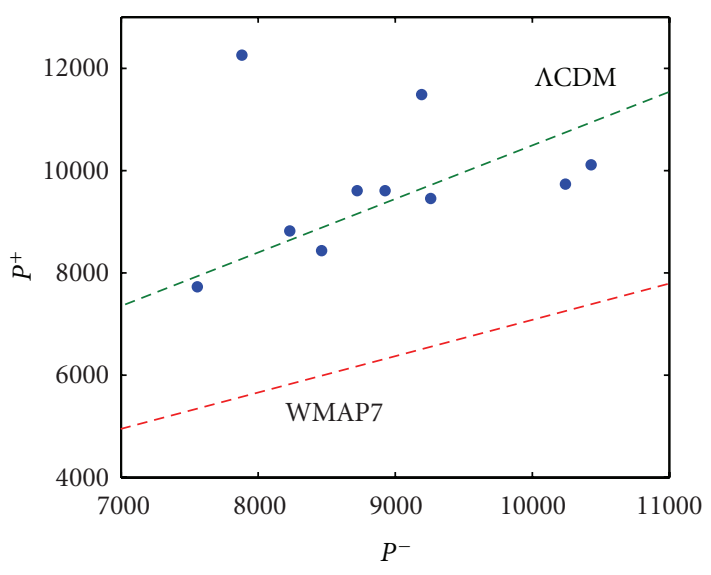

- W4

(e)

(f)

FIGURE 10: The parity asymmetry in the presence of beam asymmetry: the dots denote $\left(P^{+}, P^{-}\right)$of CMB maps simulated with asymmetric beams. The dashed lines are plotted with slopes corresponding to the $P^{+} / P^{-}$of $\Lambda$ CDM model (red) and WMAP7 data (green), respectively. The alphanumeric values at the lower right corner denote the frequency band and D/A channel. 


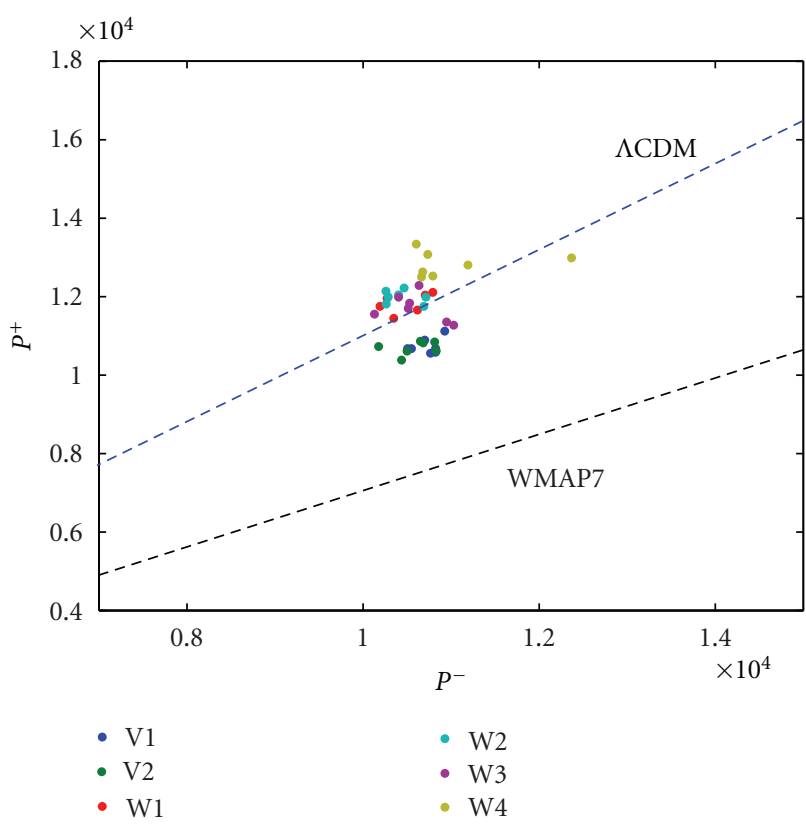

Figure 11: $P^{+}$and $P^{-}$of the WMAP team's simulation for $\mathrm{V}$ and $\mathrm{W}$ band data.

primordial perturbation $\Phi(\mathbf{k})$. Using (2), we may show the decomposition coefficients of CMB anisotropy are given by

$$
\begin{aligned}
a_{l m}= & \frac{(-\imath)^{l}}{2 \pi^{2}} \int_{0}^{\infty} d k \int_{0}^{\pi} d \theta_{\mathbf{k}} \sin \theta_{\mathbf{k}} \int_{0}^{2 \pi} d \phi_{\mathbf{k}} \Phi(\mathbf{k}) g_{l}(k) Y_{l m}^{*}(\widehat{\mathbf{k}}), \\
= & \frac{(-\imath)^{l}}{2 \pi^{2}} \int_{0}^{\infty} d k \int_{0}^{\pi} d \theta_{\mathbf{k}} \sin \theta_{\mathbf{k}} \int_{0}^{\pi} d \phi_{\mathbf{k}} g_{l}(k) \\
& \times\left(\Phi(\mathbf{k}) Y_{l m}^{*}(\hat{\mathbf{k}})+\Phi(-\mathbf{k}) Y_{l m}^{*}(-\hat{\mathbf{k}})\right), \\
= & \frac{(-\imath)^{l}}{2 \pi^{2}} \int_{0}^{\infty} d k \int_{0}^{\pi} d \theta_{\mathbf{k}} \sin \theta_{\mathbf{k}} \int_{0}^{\pi} d \phi_{\mathbf{k}} g_{l}(k) Y_{l m}^{*}(\hat{\mathbf{k}}) \\
& \times\left(\Phi(\mathbf{k})+(-1)^{l} \Phi^{*}(\mathbf{k})\right),
\end{aligned}
$$

where we used the reality conditions $\Phi(-\mathbf{k})=\Phi^{*}(\mathbf{k})$ and $Y_{l m}(-\hat{\mathbf{n}})=(-1)^{l} Y_{l m}(\hat{\mathbf{n}})$. Using (11), it is trivial to show, for the odd number multipoles $l=2 n-1$,

$$
\begin{aligned}
a_{l m}= & -\frac{(-\imath)^{l-1}}{\pi^{2}} \int_{0}^{\infty} d k \int_{0}^{\pi} d \theta_{\mathbf{k}} \sin \theta_{\mathbf{k}} \\
& \times \int_{0}^{\pi} d \phi_{\mathbf{k}} g_{l}(k) Y_{l m}^{*}(\hat{\mathbf{k}}) \operatorname{Im}[\Phi(\mathbf{k})],
\end{aligned}
$$

and, for even number multipoles $l=2 n$,

$$
\begin{aligned}
a_{l m}= & \frac{(-\imath)^{l}}{\pi^{2}} \int_{0}^{\infty} d k \int_{0}^{\pi} d \theta_{\mathbf{k}} \sin \theta_{\mathbf{k}} \\
& \times \int_{0}^{\pi} d \phi_{\mathbf{k}} g_{l}(k) Y_{l m}^{*}(\widehat{\mathbf{k}}) \operatorname{Re}[\Phi(\mathbf{k})] .
\end{aligned}
$$

It should be noted that the above equations are simple reformulation of (2). From (11) and (12), we may see that the odd-parity preference might be produced, provided

$$
|\operatorname{Re}[\Phi(\mathbf{k})]| \ll|\operatorname{Im}[\Phi(\mathbf{k})]| \quad\left(k \lesssim \frac{22}{\eta_{0}}\right),
$$

where $\eta_{0}$ is the present conformal time. Taking into account the reality condition $\Phi(-\mathbf{k})=\Phi^{*}(\mathbf{k})$, we may show primordial perturbation in real space is given by

$$
\begin{aligned}
\Phi(\mathbf{x})= & 2 \int_{0}^{\infty} d k \int_{0}^{\pi} d \theta_{\mathbf{k}} \sin \theta_{\mathbf{k}} \int_{0}^{\pi} d \phi_{\mathbf{k}} \\
& \times(\operatorname{Re}[\Phi(\mathbf{k})] \cos (\mathbf{k} \cdot \mathbf{x})-\operatorname{Im}[\Phi(\mathbf{k})] \sin (\mathbf{k} \cdot \mathbf{x})) .
\end{aligned}
$$

Noting (13) and (14), we find our primordial Universe may possess odd-parity preference on large scales $\left(2 / \eta_{0} \lesssim\right.$ $\left.k \lesssim 22 / \eta_{0}\right)$. The odd-parity preference of our primordial Universe violates large-scale translational invariance in all directions. However, it is not in direct conflict with the current data on observable Universe (i.e., WMAP CMB data), though it may seem intriguing. Considering (13) and (14), we find this effect will be manifested on the scales larger than $2 \pi \eta_{0} / 22 \approx 4 \mathrm{Gpc}$. However, it will be difficult to observe such large-scale effects in non-CMB observations. If the odd-parity preference is indeed cosmological, it indicates we are at a special place in the Universe, which may sound intriguing. However, it should be noted that the invalidity of the Copernican Principle such as our living near the center of void had been previously proposed in different context $[86,87]$.

Depending on the type of cosmological origins (e.g., topology, features in primordial power spectrum and (13)), distinct anomalies are predicted in polarization power spectrum. Therefore, polarization maps of large-sky coverage (i.e., low multipoles) will allow us to remove degeneracy and figure a cosmological origin, if the parity asymmetry is indeed cosmological.

\section{Phase of Even and Odd Multipole Data}

The decomposition coefficients $a_{l m}$ of CMB anisotropy, which are briefly discussed in Section 2, are equivalently written as

$$
a_{l m}=\left|a_{l m}\right| \exp \left(i \phi_{l m}\right) .
$$

Given a Gaussian model, we expect that the amplitudes $\left|a_{l, m}\right|$ and the phase $\phi_{l m}$ follow the Rayleigh distribution and a uniform distribution $[0,2 \pi]$, respectively $[3,4$, 88]. Therefore, the phase information provides additional information on the statistical properties and hence useful test on Gaussianity. Noting this, we have investigated the phases and compared those of even and odd multipole data. For 
the analysis, we are going to use the following trigonometric moments:

$$
\begin{aligned}
& \&(l)=\frac{1}{2 l+1} \sum_{m=-l}^{l} \sin \left(\varphi_{l m}\right), \\
& \mathcal{C}(l)=\frac{1}{2 l+1} \sum_{m=-l}^{l} \cos \left(\varphi_{l m}\right) .
\end{aligned}
$$

Using the trigonometric moments, we may estimate the mean angle $\Theta(l)$ as follows:

$$
\Theta(l)=\arctan \left(\frac{\delta(l)}{\mathcal{C}(l)}\right),
$$

where the information of the $\mathrm{CMB}$ phases is condensed into a single mean angle for an individual multipole. Further details on the procedures above can be found in [89]. Given a Gaussian random Universe, we would expect the mean angles of each multipoles to follow a uniform distribution $(-\pi \leq \Theta \leq \pi)$ [90]. In order to investigate the even and odd multipole data, respectively, we will use the following statistics:

$$
\begin{gathered}
r_{s}^{ \pm}\left(l_{\max }\right)=\sum_{l=2}^{l_{\max }} \sin \Theta^{ \pm}(l), \\
r_{c}^{ \pm}\left(l_{\max }\right)=\sum_{l=3}^{l_{\max }} \cos \Theta^{ \pm}(l), \\
R^{ \pm}\left(l_{\max }\right)=\frac{1}{l_{\max }-2}\left(\left[r_{s}^{ \pm}\left(l_{\max }\right)\right]^{2}+\left[r_{c}^{ \pm}\left(l_{\max }\right)\right]^{2}\right),
\end{gathered}
$$

where we imply that the quantities of + and - are associated with the even and odd multipole data, respectively. In the theory of statistical analysis of circular data, the statistic $R$ is widely used (refer to [91] for details). After a simple algebra on (22), we may easily show

$$
\begin{aligned}
R^{ \pm}\left(l_{\max }\right)= & \frac{1}{l_{\max }-2} \sum_{l l^{\prime}}^{l} \cos \left(\Theta^{ \pm}(l)-\Theta^{ \pm}\left(l^{\prime}\right)\right) \\
= & \frac{1}{2}\left(\delta_{l_{\max }, 2 n}+\frac{l_{\max }-1}{l_{\max }-2} \delta_{l_{\max }, 2 n+1}\right) \\
& +\frac{1}{l_{\max }-2} \sum_{l, l^{\prime} \neq l}^{l} \cos \left(\Theta^{ \pm}(l)-\Theta^{ \pm}\left(l^{\prime}\right)\right) .
\end{aligned}
$$

If the mean angles are correlated among distinct multipoles, the second term in (19) is given by $\sim l^{-2}$, and thus $R^{ \pm}$ asymptotically approaches $1 / 2$ [35]. On the other hand, if the mean angles of distinct multipoles are similar to each other (i.e., $\left.\cos \left(\Theta^{ \pm}(l)-\Theta^{ \pm}\left(l^{\prime}\right)\right) \sim \pm 1\right)$, the second term in (19) is comparable to the first term, and thus $R^{ \pm}$asymptotically approaches 1 .

In order to investigate the correlation of mean angles, we have estimated $\cos (\Theta(l)-\Theta(l+\Delta l))$. For $\Delta l=1$ and $\Delta l=$ 2 , we find several unusual alignment for various multipoles, where the unusual alignment is found in Galactic coordinate
TABLE 3: Mean angle correlation of WMAP data in Galactic coordinate.

\begin{tabular}{lc}
\hline$l$ & $\cos (\Theta(l)-\Theta(l+1))$ \\
\hline 2 & 0.9714 \\
18 & 0.9947 \\
28 & 0.9978 \\
33 & 0.9477 \\
36 & 0.9299 \\
38 & 0.9485 \\
\hline
\end{tabular}

TABLE 4: Mean angle correlation of WMAP data in Ecliptic coordinate.

\begin{tabular}{lc}
\hline$l$ & $\cos (\Theta(l)-\Theta(l+2))$ \\
\hline 5 & 0.9986 \\
23 & 0.9995 \\
33 & 0.9998 \\
34 & 0.9999 \\
\hline
\end{tabular}

and Ecliptic coordinate, respectively, for $\Delta l=1$ and $\Delta l=2$. In Tables 3 and 4 , we show the values of $\cos (\Theta(l)-\Theta(l+1))$ and $\cos (\Theta(l)-\Theta(l+2))$.

In Figure 12, we plot $r^{ \pm}(l)$ of the WMAP team's ILC map, which are estimated in Galactic coordinate and Ecliptic coordinate, respectively. As shown in the figure, the mean angles of the even and odd are distinct from each other, which is significant at $\sim 3 \sigma$ level. In Figure 13, we show $R^{ \pm}(l)$ and their inverse for various values of $l$, which are estimated in Galactic coordinate. From this figure, we can see that a major contribution to the $R^{-}(l)$ comes from the multipole $5 \leq l \leq 30$, where the $R^{-}(l)$ exceeds the $2 \sigma$. The even multipoles, in contrast with the odd ones, show noticeably small values of the $R^{+}(l)$-parameter, which indicate the correlations between the mean angles. It should be noted that values of $R^{-}(l)$ and $R^{+}(l)$ are expected to be around $\sim 0.5$. Therefore, unusually high or small values as those of WMAP data indicate unusual correlation of mean angles, and the deviation from statistically isotropic Gaussian Universe.

\section{Antisymmetry of the Octupole Component}

Using (1) and the reality condition $a_{l m}=a_{l-m}^{*}$, we may easily show that a whole-sky CMB anisotropy pattern is given by

$$
\begin{aligned}
T(\theta, \phi)= & \sum_{l} a_{l 0} N_{l 0} P_{l}(\cos \theta)+2 \sum_{l} \sum_{m \geq 1} N_{l m} P_{l}^{m}(\cos \theta) \\
& \times\left(\operatorname{Re}\left[a_{l m}\right] \cos (m \phi)-\operatorname{Im}\left[a_{l m}\right] \sin (m \phi)\right),
\end{aligned}
$$

where $P_{l}(\cos \theta)$ and $P_{l}^{m}(\cos \theta)$ are the Legendre polynomials and the associated Legendre polynomials, respectively, and

$$
N_{l m}=(-1)^{m} \sqrt{\frac{(2 l+1)(l-m)}{4 \pi(l+m) !}} .
$$




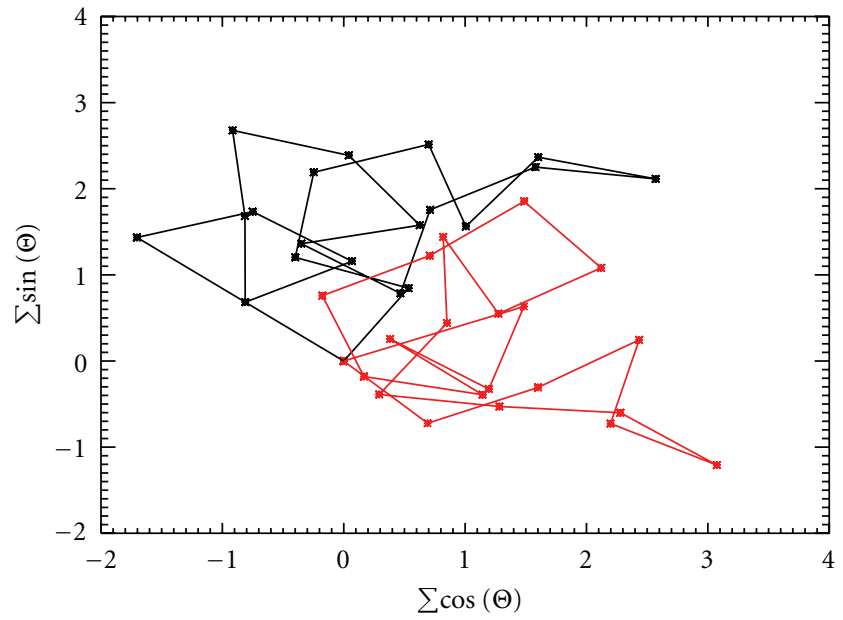

(a)

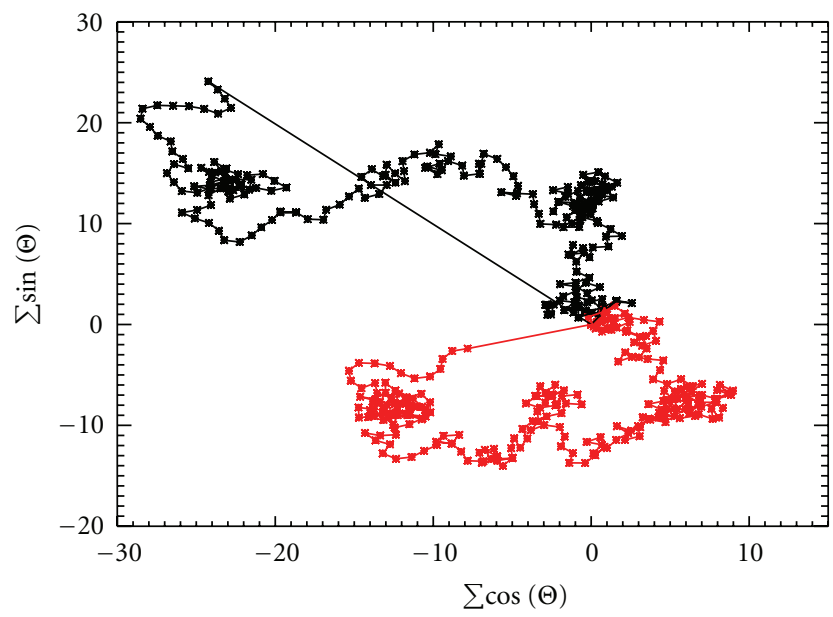

(b)

Figure 12: (a) The parameter $r^{-}(l)$ (the black dots) for odd multipoles and $r^{+}(l)$ (the red dots) for even ones in Galactic coordinates. The mean angles are estimated from the first 40 multipoles. (b) the same as the (a), but for ecliptic coordinates.

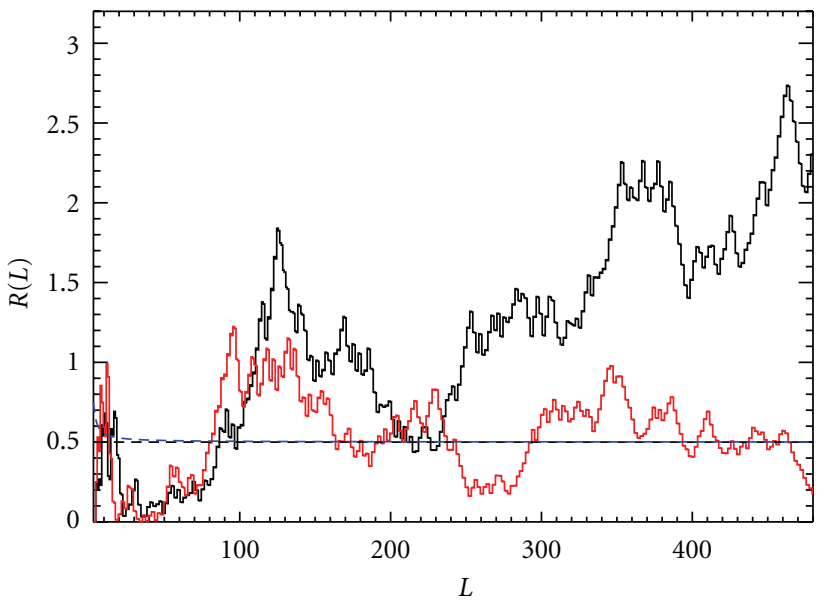

Figure 13: The parameters $R^{-}(l)$ (top panel, the black line) for odd multipoles and $R^{+}(l)$ (top panel, the red line) for even multipoles. The black dash line is the asymptotic $R^{+}(l=$ even $)=0.5$, the blue dash line is for $R^{-}(l=$ odd $)=(l-1) / 2(l-2)$.

In addition to the parity operation (i.e., $\mathbf{n} \rightarrow-\mathbf{n}$ ) discussed previously, we may consider the following coordinate inversion: $(\theta, \phi) \rightarrow(\pi-\theta,-\phi)$. In a similar way to the investigation on the parity asymmetry, we construct a symmetric and antisymmetric part under the coordinate inversion $(\theta, \phi) \rightarrow(\pi-\theta, 2 \pi-\phi)$ as follows:

$$
T^{ \pm}(\theta, \phi)=\frac{T(\theta, \phi) \pm T(\pi-\theta,-\phi)}{2} .
$$

Using (20), we may easily show the symmetric and antisymmetric parts are given by

$$
\begin{aligned}
T^{ \pm}(\theta, \phi)= & \sum_{l} N_{l 0} P_{l}(\cos \theta) \operatorname{Re}\left[a_{l 0}\right] \frac{1 \pm(-1)^{l}}{2} \\
& +2 \sum_{l} \sum_{m \geq 1} N_{l m} P_{l}^{m}(\cos \theta) \\
& \times\left(\operatorname{Re}\left[a_{l m}\right] \frac{1 \pm(-1)^{l+m}}{2}\right. \\
& \times \cos (m \phi) \operatorname{Im}\left[a_{l m}\right] \frac{1 \mp(-1)^{l+m}}{2} \\
& -\sin (m \phi)) .
\end{aligned}
$$

As obvious in (23), the symmetric part gets contribution only from $\operatorname{Re}\left[a_{l m}\right]$ of $l+m=$ even and $\operatorname{Im}\left[a_{l m}\right]$ of $l+m=$ odd. On the other hand, the antisymmetric part gets contribution only from $\operatorname{Re}\left[a_{l m}\right]$ of $l+m=$ odd and $\operatorname{Im}\left[a_{l m}\right]$ of $l+m=$ even. Noting this, we have estimated the ratio of $l+m=$ odd and $l+m=$ even components for the real and imaginary parts, where we used the WMAP 7 year ILC map. Our estimation shows the ratio $\operatorname{Im}\left[a_{33}\right] / \operatorname{Im}\left[a_{32}\right]$ is unusually high, which requires the chance of 6-in-1000 level. In Figure 14, we show the octupole components of the WMAP7 ILC map (top), $T^{+}$(middle), and $T^{-}$(bottom). From Figure 14, we may see that the octupole components of the WMAP7 ILC map show antisymmetric pattern for the inversion $(\theta, \phi) \rightarrow(\pi-\theta,-\phi)$, where the center of the images corresponds to the coordinate $(\theta=0, \phi=0)$. 


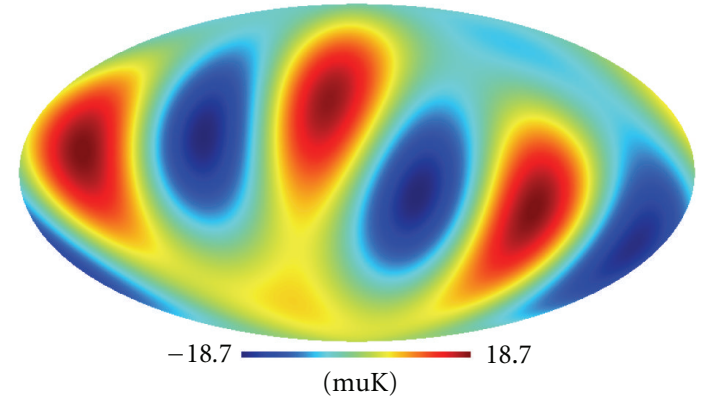

(a)

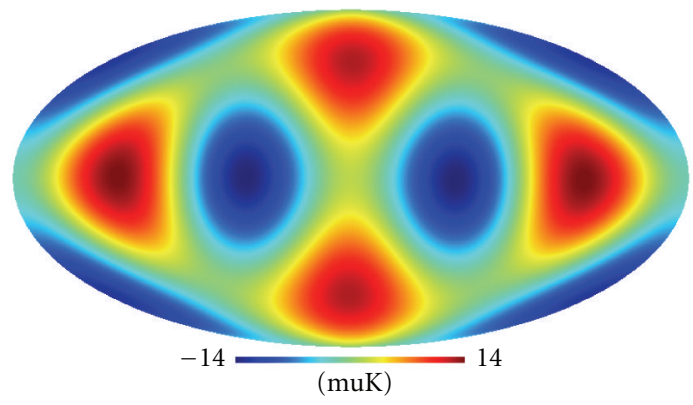

(b)

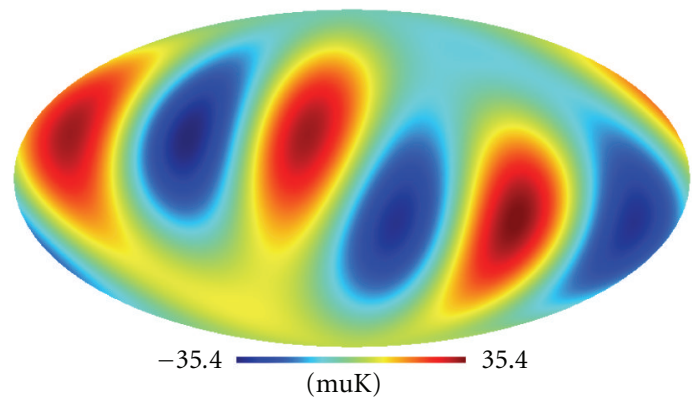

(c)

FIGURE 14: The octupole components of the WMAP7 ILC map (a), $T_{3}^{+}(\theta, \phi)(\mathrm{b})$, and $T_{3}^{-}(\theta, \phi)(\mathrm{c})$.

\section{Parametric Tension between Even and Odd Multipole Data}

As discussed previously, there is the power contrast between even and odd multipoles of WMAP TT power spectrum $[20,37-39,45]$. At lowest multipoles $(2 \leq l \leq 22)$, there is odd multipole preference (i.e., power excess in odd multipoles and deficit in even multipoles) [37-39, 45], and additionally even multipole preference at intermediate multipoles $(200 \leq l \leq 400)$ [20]. For TE correlation, we have also found odd multipole preference at $(100 \lesssim$ $l \lesssim 200)$ and even multipole preference at $(200 \lesssim l \lesssim$ 400), though its statistical significance is not high enough, due to low signal-to-noise ratio of polarization data. Not surprisingly, these power contrast anomalies are explicitly associated with the angular power spectrum data, which are mainly used to fit cosmological models. Having noted this, we have investigated whether the even (odd) multipole data set is consistent with the concordance model. For a cosmological model, we have considered $\Lambda \mathrm{CDM}+\mathrm{SZ}$ effect + weak-lensing, where cosmological parameters are $\lambda \in$ $\left\{\Omega_{\mathrm{b}}, \Omega_{\mathrm{c}}, \tau, n_{\mathrm{s}}, A_{\mathrm{s}}, A_{\mathrm{sz}}, H_{0}\right\}$. For data constraints, we have used the WMAP 7-year TT and TE power spectrum data, which have been estimated from the ILC map, and cut-sky $\mathrm{V}$ and $\mathrm{W}$ band maps [6]. Hereafter, we shall denote WMAP CMB data of whole, even and odd multipoles by $D_{0}, D_{2}$, and $D_{3}$, respectively. We like to stress that even/odd multipole splitting is made for TT and TE power spectrum up to the multipoles of WMAP sensitivity (i.e., $l \leq 1200$ for TT and $l \leq 800$ for TE). Using CosmoMC with the modified WMAP likelihood code, we have explored the parameter space on a MPI cluster with 6 chains [6, 92-94]. For the convergence criterion, we have adopted the Gelman and Rubin's “variance of chain means" and set the $\mathrm{R}-1$ statistic to 0.03 for stopping criterion $[95,96]$.

In Figure 15, we show the marginalized likelihood of parameters, which are obtained from the run of a CosmoMC with $D_{0}, D_{2}$, and $D_{3}$, respectively. In Table 5 , we show the best-fit parameters and $1 \sigma$ confidence intervals, where $\lambda_{2}$ and $\lambda_{3}$ denote the best-fit values of $D_{2}$ and $D_{3}$, respectively. The parameter set $\lambda_{0}$ is the best-fit values of whole data $D_{0}$ and accordingly corresponds to the WMAP concordance model. As shown in Figure 15 and Table 5, we find nonnegligible tension especially in parameters of primordial power spectrum. It is worth to note that the best-fit spectral index of even multipole data (i.e., $D_{2}$ ) is close to a flat spectrum (i.e., $n_{s}=1$ ), while the result from the whole data rules out the flat spectrum by more than $2 \sigma$.

There is a likelihood-ratio test, which allows us to determine the rejection region of an alternative hypothesis, given a null hypothesis [97-100]. By setting sets of parameters to a null hypothesis and an alternative hypothesis, we may investigate whether two sets of parameters are consistent with each other. To be specific, we have evaluated the following in order to assess parametric tension:

$$
\frac{\mathcal{L}\left(\lambda_{j} \mid D_{\mathrm{i}}\right)}{\mathcal{L}\left(\lambda_{i} \mid D_{i}\right)},
$$




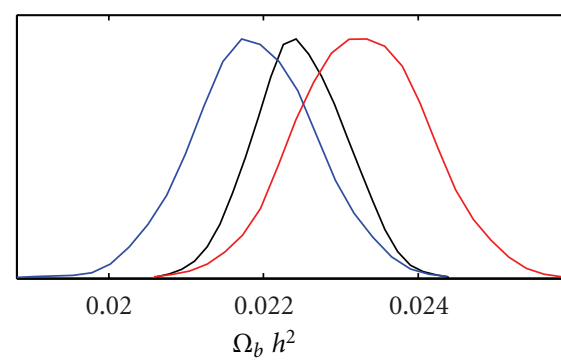

(a)

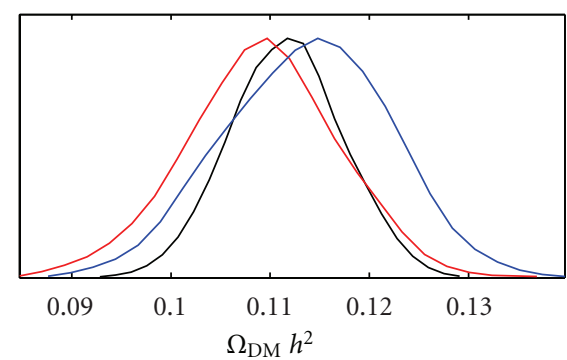

(b)

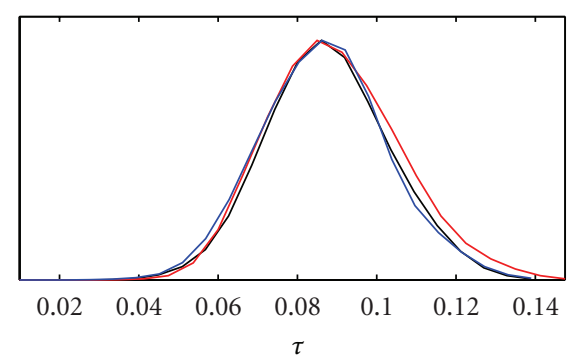

(c)

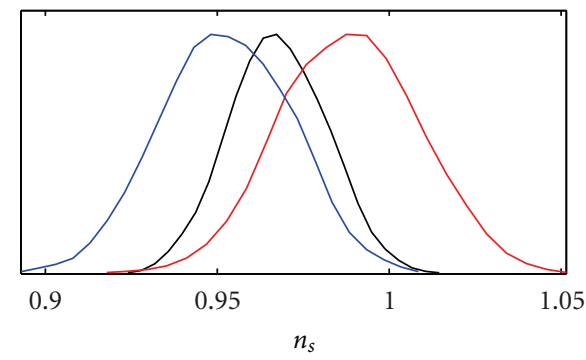

(d)

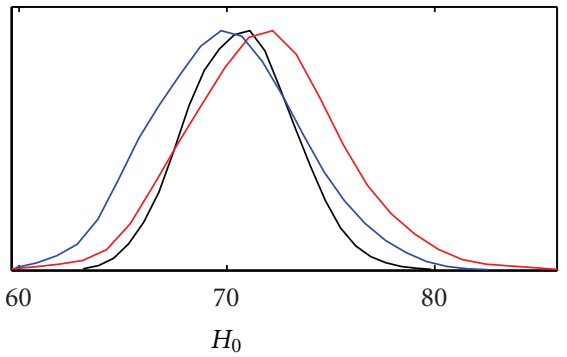

(f)

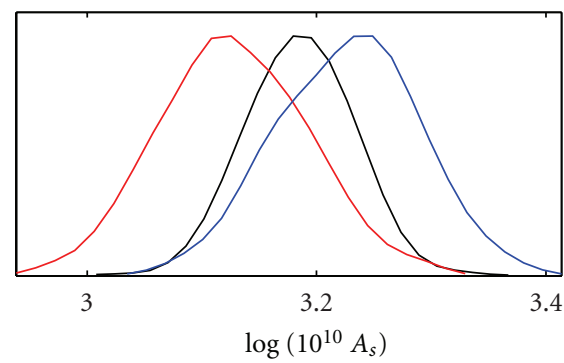

(e)

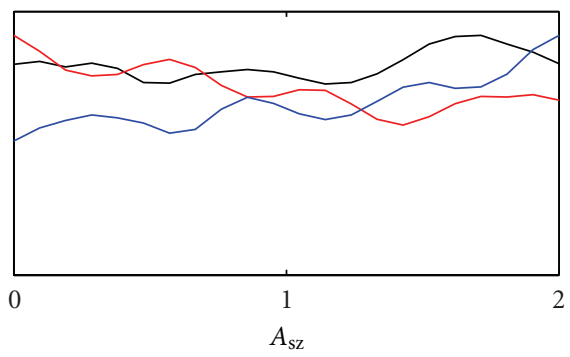

- All multipoles

Even multipoles

— Odd multipoles

(g)

Figure 15: Marginalized likelihood of cosmological parameters ( $\Lambda \mathrm{CDM}+\mathrm{sz}+$ lens), given whole or even (odd) multipole data.

TABLE 5: Cosmological parameters $(\Lambda \mathrm{CDM}+\mathrm{sz}+$ lens $)$.

\begin{tabular}{lccc}
\hline & $\lambda_{0}$ & $\lambda_{2}$ & $\lambda_{3}$ \\
\hline$\Omega_{b} h^{2}$ & $0.0226 \pm 0.0006$ & $0.0231 \pm 0.0008$ & $0.0217 \pm 0.0008$ \\
$\Omega_{c} h^{2}$ & $0.112 \pm 0.006$ & $0.109 \pm 0.008$ & $0.115 \pm 0.008$ \\
$\tau$ & $0.0837 \pm 0.0147$ & $0.0913 \pm 0.0157$ & $0.0859 \pm 0.015$ \\
$n_{s}$ & $0.964 \pm 0.014$ & $0.989 \pm 0.02$ & $0.949 \pm 0.019$ \\
$\log \left[10^{10} A_{s}\right]$ & $3.185 \pm 0.047$ & $3.132 \pm 0.065$ & $3.239 \pm 0.062$ \\
$H_{0}$ & $70.53 \pm 2.48$ & $71.73 \pm 3.59$ & $69.68 \pm 3.47$ \\
$A_{s z}$ & $1.891_{-1.891}^{+0.109}$ & $0.169_{-0.169}^{+1.831}$ & $0.89_{-0.89}^{+1.11}$ \\
\hline
\end{tabular}

where parameter sets $\lambda_{i}$ and $\lambda_{j}$ correspond to a null hypothesis and an alternative hypothesis, respectively. In Table 6, we show the likelihood ratio, where the quantities used for the numerator and denominator are indicated in the uppermost row and leftmost column. As shown by $\mathcal{L}\left(\lambda_{0} \mid D_{2}\right) / \mathcal{L}\left(\lambda_{2} \mid D_{2}\right)$ and $\mathcal{L}\left(\lambda_{0} \mid D_{3}\right) / \mathcal{L}\left(\lambda_{3} \mid D_{3}\right)$, the WMAP concordance model (i.e., $\left.\lambda_{0}\right)$ does not make a good fit for
TABLE 6: The likelihood ratio: $\Lambda \mathrm{CDM}+\mathrm{sz}+$ lens.

\begin{tabular}{cccc}
\hline & $\mathcal{L}\left(\lambda_{0} \mid D_{0}\right)$ & $\mathcal{L}\left(\lambda_{2} \mid D_{0}\right)$ & $\mathcal{L}\left(\lambda_{3} \mid D_{0}\right)$ \\
$\mathcal{L}\left(\lambda_{0} \mid D_{0}\right)$ & 1 & 0.076 & 0.0099 \\
\hline & $\mathcal{L}\left(\lambda_{0} \mid D_{2}\right)$ & $\mathcal{L}\left(\lambda_{2} \mid D_{2}\right)$ & $\mathcal{L}\left(\lambda_{3} \mid D_{2}\right)$ \\
$\mathcal{L}\left(\lambda_{2} \mid D_{2}\right)$ & 0.16 & 1 & $2 \times 10^{-4}$ \\
\hline & $\mathcal{L}\left(\lambda_{0} \mid D_{3}\right)$ & $\mathcal{L}\left(\lambda_{2} \mid D_{3}\right)$ & $\mathcal{L}\left(\lambda_{3} \mid D_{3}\right)$ \\
$\mathcal{L}\left(\lambda_{3} \mid D_{3}\right)$ & 0.16 & 0.0022 & 1 \\
\hline
\end{tabular}

even (odd) multipole data set. Besides, there exists significant tension between two data subsets, as indicated by very small values of $\mathcal{L}\left(\lambda_{3} \mid D_{2}\right) / \mathcal{L}\left(\lambda_{2} \mid D_{2}\right)$ and $\mathcal{L}\left(\lambda_{2} \mid D_{3}\right) / \mathcal{L}\left(\lambda_{3} \mid D_{3}\right)$. The parameter likelihood, except for $A_{\mathrm{sz}}$, follows the shape of Gaussian functions, as shown in Figure 15. For a likelihood of Gaussian shape, the likelihood ratios 0.1353 and 0.0111 correspond to $2 \sigma$ and $3 \sigma$ significance level, respectively. From Table 6 , we may see most of the ratio indicates $\sim 2 \sigma$ tension or even higher. 


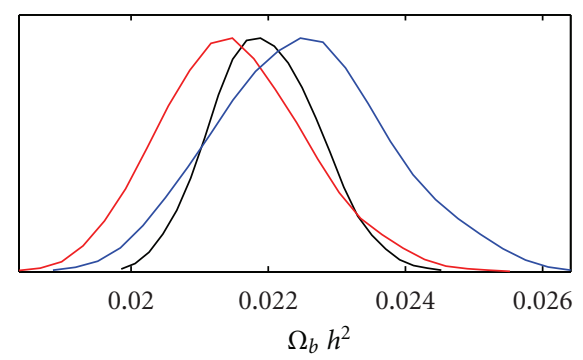

(a)

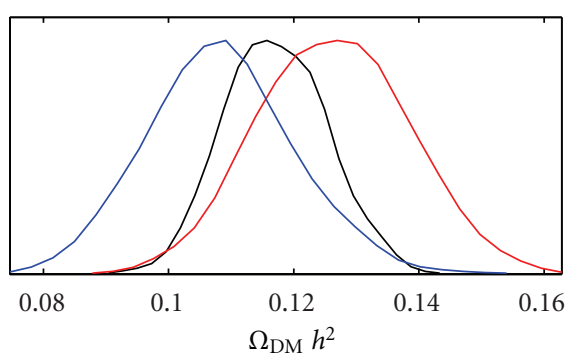

(b)

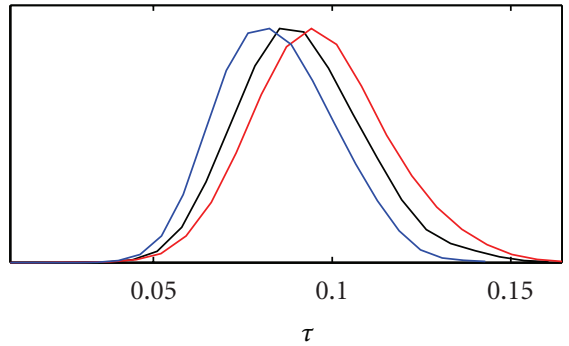

(c)

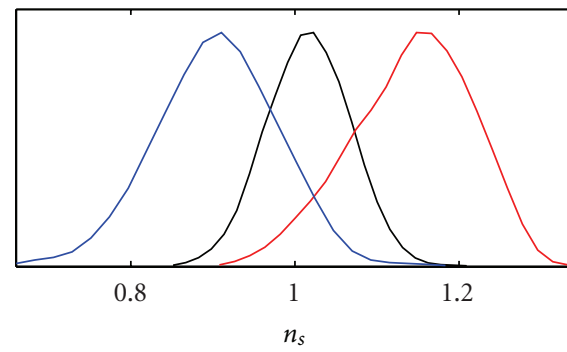

(d)

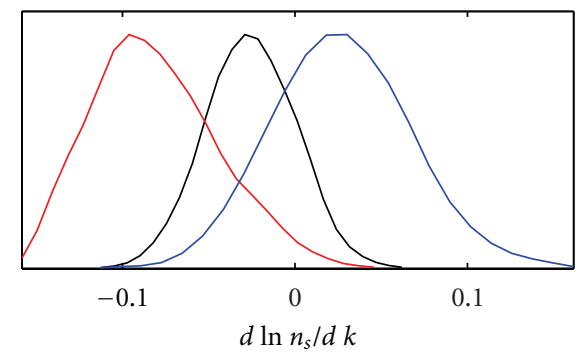

(e)
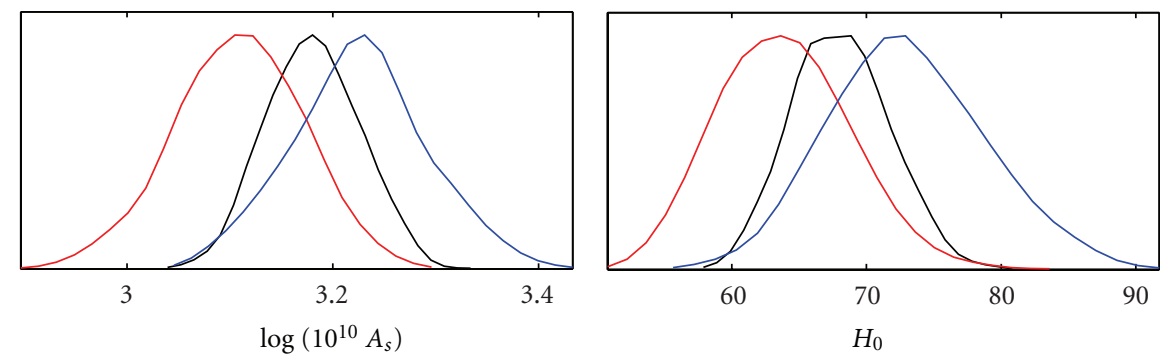

- All multipoles

_ Even multipoles

— Odd multipoles

(f)

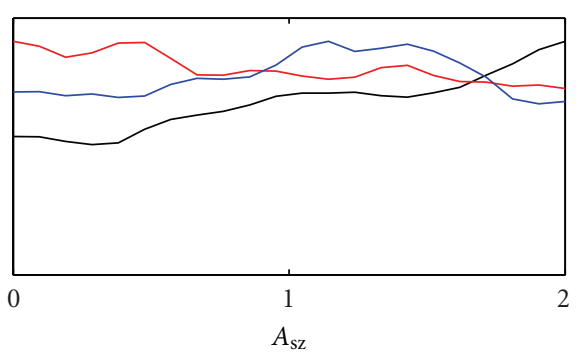

_ All multipoles

_ Even multipoles

— Odd multipoles

(h)

Figure 16: Marginalized likelihood of cosmological parameters $(\Lambda \mathrm{CDM}+\mathrm{sz}+$ lens + run $)$, given whole, even, and odd multipole data, respectively.

As discussed previously, the tension is highest in parameters of primordial power spectrum, which may be an indication of missing parameters in primordial power spectrum (e.g., a running spectral index). Therefore, we have additionally considered a running spectral index $d n_{s} / d \ln k$ and repeated our investigation. Surprisingly, we find tension increases to even a higher level. We show the marginalized parameter likelihoods and the likelihood ratios in Figure 16 and Table 7, where we find tension is also highest in the primordial power spectrum parameters. These tensions 
TABLE 7: The likelihood ratio: $\Lambda \mathrm{CDM}+\mathrm{sz}+$ lens + run.

\begin{tabular}{cccc}
\hline & $\mathcal{L}\left(\lambda_{0} \mid D_{0}\right)$ & $\mathcal{L}\left(\lambda_{2} \mid D_{0}\right)$ & $\mathcal{L}\left(\lambda_{3} \mid D_{0}\right)$ \\
$\mathcal{L}\left(\lambda_{0} \mid D_{0}\right)$ & 1 & $3.5 \times 10^{-4}$ & 0.0078 \\
\hline & $\mathcal{L}\left(\lambda_{0} \mid D_{2}\right)$ & $\mathcal{L}\left(\lambda_{2} \mid D_{2}\right)$ & $\mathcal{L}\left(\lambda_{3} \mid D_{2}\right)$ \\
$\mathcal{L}\left(\lambda_{2} \mid D_{2}\right)$ & 0.06 & 1 & $2.3 \times 10^{-5}$ \\
\hline & $\mathcal{L}\left(\lambda_{0} \mid D_{3}\right)$ & $\mathcal{L}\left(\lambda_{2} \mid D_{3}\right)$ & $\mathcal{L}\left(\lambda_{3} \mid D_{3}\right)$ \\
$\mathcal{L}\left(\lambda_{3} \mid D_{3}\right)$ & 0.042 & $5.8 \times 10^{-7}$ & 1 \\
\hline
\end{tabular}

indicate there is either unaccounted contamination or the failure of the assumed cosmological model (i.e., the flat $\Lambda$ CDM model).

\section{Lack of Angular Correlation in the WMAP Data}

Given CMB anisotropy data, we may estimate two point angular correlation as follows:

$$
C(\theta)=T\left(\widehat{\mathbf{n}}_{1}\right) T\left(\hat{\mathbf{n}}_{2}\right),
$$

where $\theta=\cos ^{-1}\left(\hat{\mathbf{n}}_{1} \cdot \hat{\mathbf{n}}_{2}\right)$. Using (1) and (3), we may easily show that the expectation value of the correlation is given by [101]:

$$
\langle C(\theta)\rangle=\sum_{l} \frac{2 l+1}{4 \pi} W_{l} C_{l} P_{l}(\cos \theta)
$$

where $\theta$ is a separation angle, $W_{l}$ is the window function of the observation, and $P_{l}$ is a Legendre polynomial. As shown in (26), the angular correlation $C(\theta)$ is the linear combination of angular power spectrum $C_{l}$, and therefore, possess equivalence.

In Figure 17, we show the angular correlation of the WMAP 7 year data, which are estimated, respectively, from the WMAP team's Internal Linear Combination (ILC) map, and foreground reduced maps of $\mathrm{V}$ and $\mathrm{W}$ band. In the angular correlation estimation, we have excluded the foreground-contaminated region by applying the WMAP KQ75 mask, as recommended for non-Gaussianity study [60]. In the same plot, we show the angular correlation of the WMAP concordance model [54], where the dotted line and shaded region denote the mean value and $1 \sigma$ ranges of Monte-Carlo simulations at V band. For simulation, we have made $10^{4}$ realizations with the same configuration with the WMAP data (e.g., a foreground mask, beam smoothing, and instrument noise). In order to include WMAP noise in simulation, we have subtracted one Differencing Assembly (D/A) data from another, and added it to simulations.

As shown in Figure 17, there exists nonnegligible discrepancy between the data and the theoretical prediction. Most noticeably, angular correlation of WMAP data nearly vanishes at angles larger than $\sim 60^{\circ}$, which are previously investigated by $[30,46-49]$. In the previous investigations,

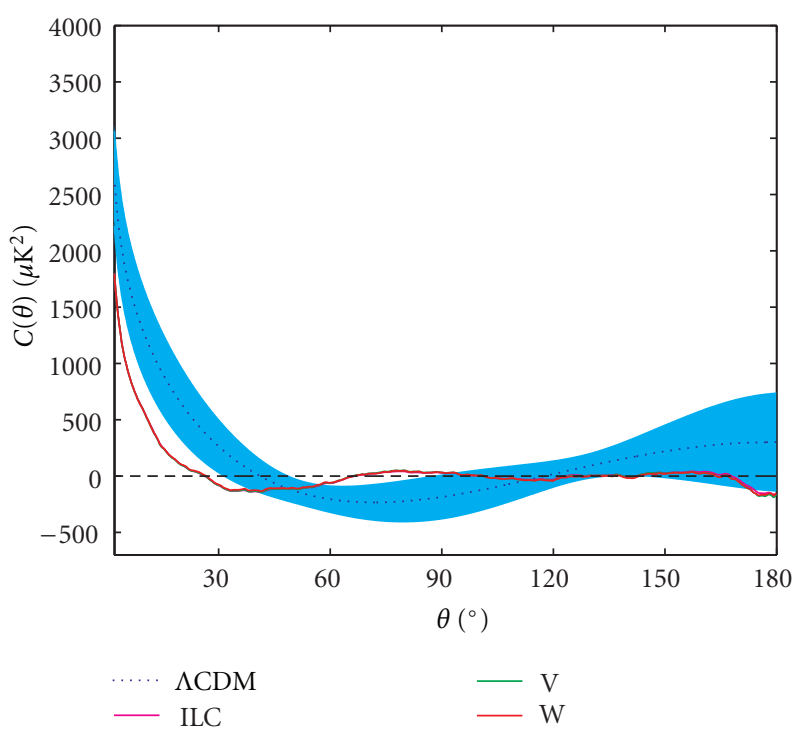

FIgURE 17: Angular correlation of CMB anisotropy: solid lines denote the angular correlation of WMAP data. Dotted line and shaded region denote the theoretical prediction and $1 \sigma$ ranges, as determined by Monte-Carlo simulations $(\Lambda \mathrm{CDM})$.

the lack of large-angle correlation has been assessed by the following statistic [30, 47-49]:

$$
S_{1 / 2}=\int_{-1}^{1 / 2}(C(\theta))^{2} d(\cos \theta) .
$$

The investigation shows the $S_{1 / 2}$ estimated from WMAP data is anomalously low, which requires the chance $\lesssim 10^{-3}$ $[30,47-50]$. Besides the lack of correlation at large angles, we may see from Figure 17 that correlation at small angles tends to be smaller than the theoretical prediction. Noting this, we have investigated the small-angle correlation with the following statistics:

$$
S_{\sqrt{3} / 2}=\int_{\sqrt{3} / 2}^{1}(C(\theta))^{2} d(\cos \theta),
$$

where the square of the correlation is integrated over small angles $\left(0 \leq \theta \leq 30^{\circ}\right)$. Therefore, the values of $S_{\sqrt{3} / 2}$ and $S_{1 / 2}$ correspond to the integrated power at small and large angles respectively. In Table 8, we show $S_{1 / 2}$ and $S_{\sqrt{3} / 2}$ of the WMAP 7-year data. Recall that the slight difference between $\mathrm{V}$ and $\mathrm{W}$ band is due to the distinct beam size, and simulations are made accordingly for each band. In the same table, we show the $P$ value, where the $P$-value denotes fractions of simulations as low as those of WMAP data. As shown in Table 8 , WMAP data have unusually low values of $S_{1 / 2}$ and $S_{\sqrt{3} / 2}$, as indicated by their $P$-value. It is worth to note that the $P$-value of $S_{\sqrt{3} / 2}$ corresponds to very high statistical significance, even though it is not as low as that of $S_{1 / 2}$. In summary, we find anomalous lack of correlation at small angles in addition to large angles.

In Figure 18, we show $S_{1 / 2}$ and $S_{\sqrt{3} / 2}$, which are estimated from the WMAP 3-, 5-, and, 7-year data, respectively. As 


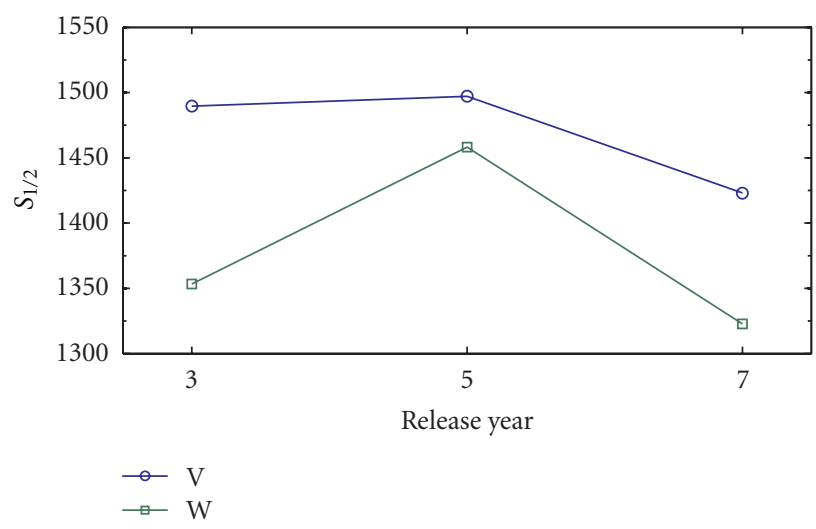

(a)

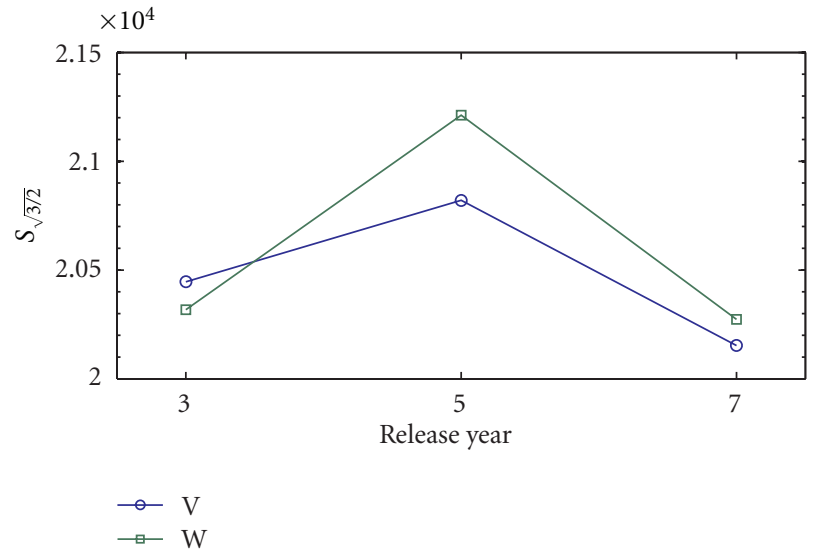

(b)

Figure 18: $S$ statistics of WMAP 3-, 5- and 7-year data.

TABLE 8: $S$ statistics of WMAP 7 year data.

\begin{tabular}{lcccc}
\hline & Band & Angles & Value $\left[\mu K^{4}\right]$ & $P$ value \\
\hline$S_{1 / 2}$ & $\mathrm{~V}$ & $60^{\circ} \leq \theta \leq 180^{\circ}$ & $1.42 \times 10^{3}$ & $8 \times 10^{-4}$ \\
$S_{1 / 2}$ & $\mathrm{~W}$ & $60^{\circ} \leq \theta \leq 180^{\circ}$ & $1.32 \times 10^{3}$ & $6 \times 10^{-4}$ \\
$S_{\sqrt{3} / 2}$ & $\mathrm{~V}$ & $0^{\circ} \leq \theta \leq 30^{\circ}$ & $2.02 \times 10^{4}$ & $3.2 \times 10^{-3}$ \\
$S_{\sqrt{3} / 2}$ & $\mathrm{~W}$ & $0^{\circ} \leq \theta \leq 30^{\circ}$ & $2.03 \times 10^{4}$ & $3.2 \times 10^{-3}$ \\
\hline
\end{tabular}

shown in Figure 18, the $S$ statistics of WMAP 7 year data are lowest, while WMAP 7 year data are believed to have more accurate calibration and less foreground contamination than earlier releases $[7,8,60]$. Therefore, we may not readily attribute the anomaly to calibration error or foregrounds.

6.1. Investigation on Noncosmological Origins. The WMAP data contain contamination from residual galactic and extragalactic foregrounds, even though we have applied the conservative KQ75 mask [60]. In order to investigate the association with residual foregrounds, we have first subtracted the foreground-reduced $\mathrm{W}$ band map from that of $\mathrm{V}$ band. This difference map mainly contains residual foregrounds of the forementioned maps with slight amount of CMB. Recall that CMB signal is not completely cancelled out, because the beam size at $\mathrm{V}$ and $\mathrm{W}$ band differs from each other. From the difference map $V(\mathbf{n})-W(\mathbf{n})$, we have obtained $S_{1 / 2}=0.31$ and $S_{\sqrt{3} / 2}=31.36$. By comparing these values with Table 8 , we may see residual foregrounds at $\mathrm{V}$ and $\mathrm{W}$ band are too small to affect the correlation power of WMAP data. In order to investigate the association of noise with the anomaly, we have produced noise maps of WMAP7 data by subtracting one Differencing Assembly (D/A) map from another $\mathrm{D} / \mathrm{A}$ data of the same frequency channel.

In Table 9, we show $S_{1 / 2}$ and $S_{\sqrt{3} / 2}$ estimated from the noise maps. Comparing Table 8 with Table 9 , we may see that the noise is not significant enough to cause the correlation anomalies of the the WMAP data. In Figure 19, we show
TABLE 9: The $S$ statistics of WMAP instrument noise in the unit of $\left(\mu K^{4}\right)$.

\begin{tabular}{lcc}
\hline Data & $S_{1 / 2}$ & $S_{\sqrt{3} / 2}$ \\
\hline V1-V2 & 0.25 & 83.94 \\
W1-W2 & 2.49 & 587.45 \\
W1-W3 & 2.18 & 664.26 \\
W1-W4 & 2.24 & 625.27 \\
W2-W3 & 2.72 & 808.32 \\
W2-W4 & 4.39 & 764.96 \\
W3-W4 & 4.39 & 764.96 \\
\hline
\end{tabular}

the values of $S_{1 / 2}$ and $S_{\sqrt{3} / 2}$ for each year and D/A data. As shown in Figure 19, the anomaly is not associated with a particular D/A channel nor a year data, but present at all year and $\mathrm{D} / \mathrm{A}$ channels, which indicates the correlation anomaly is not due to the temporal malfunctioning of a particular D/A instrument. We have also investigated simulations produced by the WMAP team, which are discussed in the Section 2. From the simulated maps, we have estimated $S_{1 / 2}$ and $S_{\sqrt{3} / 2}$, which are plotted in Figure 20. As shown in Figure 20, S statistics of simulated data are significantly higher than those of WMAP data. Therefore, the anomaly may be indeed cosmological or due to systematics, which we do not understand well.

\section{The Parity Asymmetry and the Lack of Correlation}

As shown in (26), angular power spectrum and angular correlation possess some equivalence. Noting this, we have investigated the association of the odd-parity preference with the lack of large-angle correlation, and found the odd-parity preference of the power spectrum is phenomenologically connected with the lack of large-angle correlation. Using (26) 


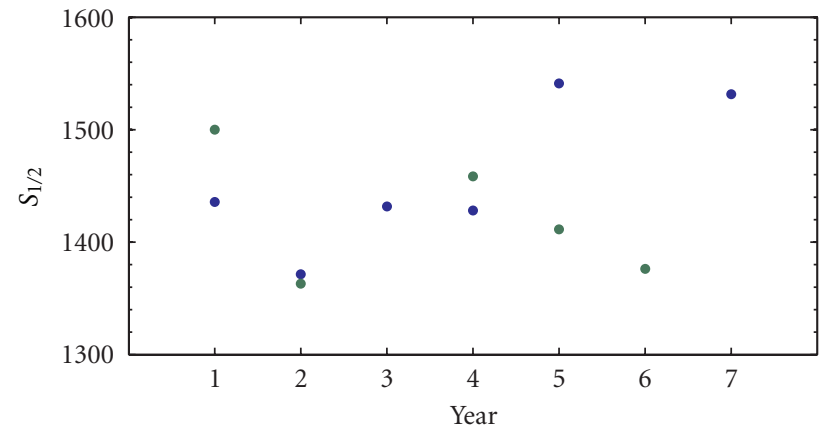

- V1

- V2

(a)

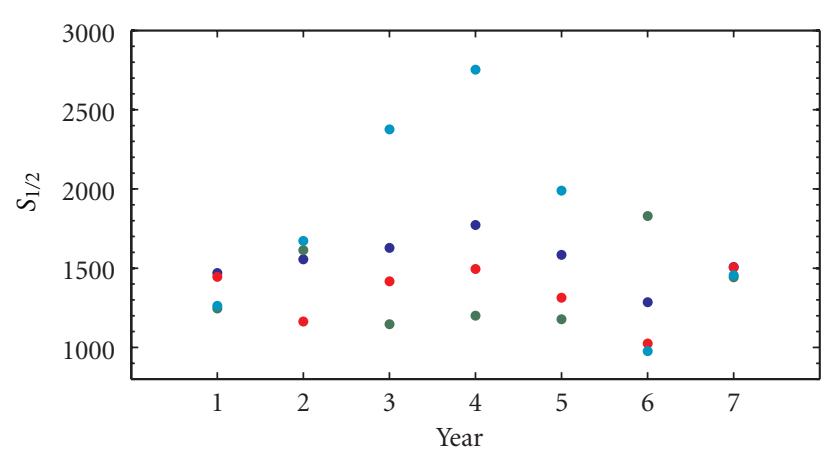

- $\mathrm{W} 1 \quad-\mathrm{W} 3$

- W2 - W4

(c)

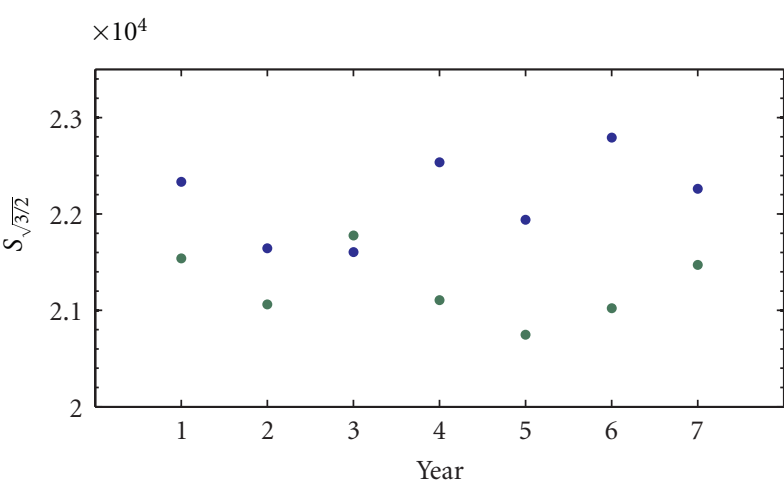

- $\mathrm{V} 1$

- V2

(b)

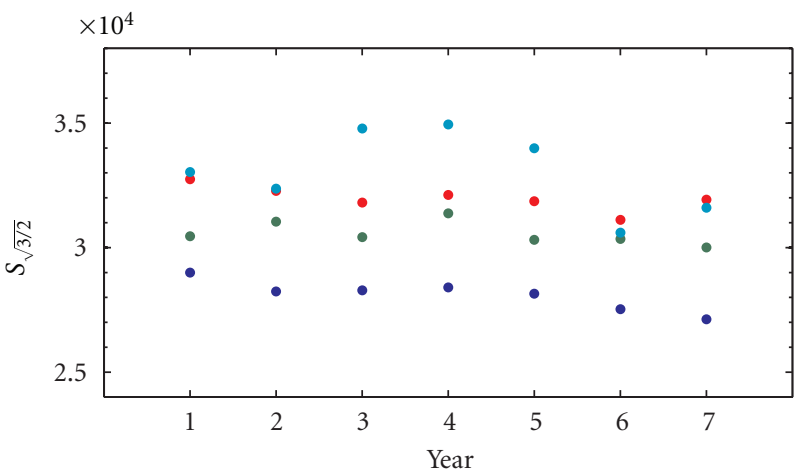

- $\mathrm{W} 1 \quad-\mathrm{W} 3$

(d)

FIGURE 19: The $S$ statistics of WMAP data at each D/A and year.

with the Sach plateau approximation (i.e., $l(l+1) C_{l} / 2 \pi \sim$ const), we find the expectation value of angular correlation is given by

$$
\begin{aligned}
C(\theta)= & \sum_{l} \frac{2 l+1}{4 \pi} W_{l} C_{l} P_{l}(\cos \theta) \\
= & \sum_{l} \frac{l(l+1) C_{l}}{2 \pi} \frac{2 l+1}{2 l(l+1)} W_{l} P_{l}(\cos \theta) \\
\approx & \alpha \sum_{l}^{l_{0}} \frac{2 l+1}{2 l(l+1)} W_{l} P_{l}(\cos \theta) \\
& +\sum_{l=l_{0}+1} C_{l} \frac{2 l+1}{4 \pi} W_{l} P_{l}(\cos \theta),
\end{aligned}
$$

where $\alpha$ is some positive constant and $l_{0}$ is a low multipole number, within which the Sach plateau approximation is valid. As discussed previously, there exists the odd multipole preference at low multipole $(2 \leq l \leq 22)$. Considering the odd multipole preference, we may show the angular correlation is given by

$$
\begin{aligned}
C(\theta) \approx & \alpha(1-\varepsilon) F(\theta)+\alpha(1+\varepsilon) G(\theta) \\
& +\sum_{l=23} C_{l} \frac{2 l+1}{4 \pi} W_{l} P_{l}(\cos \theta)
\end{aligned}
$$

where

$$
\begin{aligned}
F(\theta) & =\sum_{l}^{22} \frac{2 l+1}{2 l(l+1)} W_{l} P_{l}(\cos \theta) \cos ^{2}\left(\frac{l \pi}{2}\right), \\
G(\theta) & =\sum_{l}^{22} \frac{2 l+1}{2 l(l+1)} W_{l} P_{l}(\cos \theta) \sin ^{2}\left(\frac{l \pi}{2}\right),
\end{aligned}
$$

and $\varepsilon$ is a constant related to the parity asymmetry, which is defined to be positive for the odd parity preference and negative for the even parity preference. Accordingly, $\alpha \varepsilon(-F(\theta)+G(\theta))$ corresponds to the deviation from the standard model, due to the odd multipole preference $(2 \leq$ $l \leq 22$ ).

In Figures 21 and 22, we show $-F(\theta)+G(\theta)$ and the angular correlation of the standard model (i.e., $\varepsilon=0$ ). Let us 

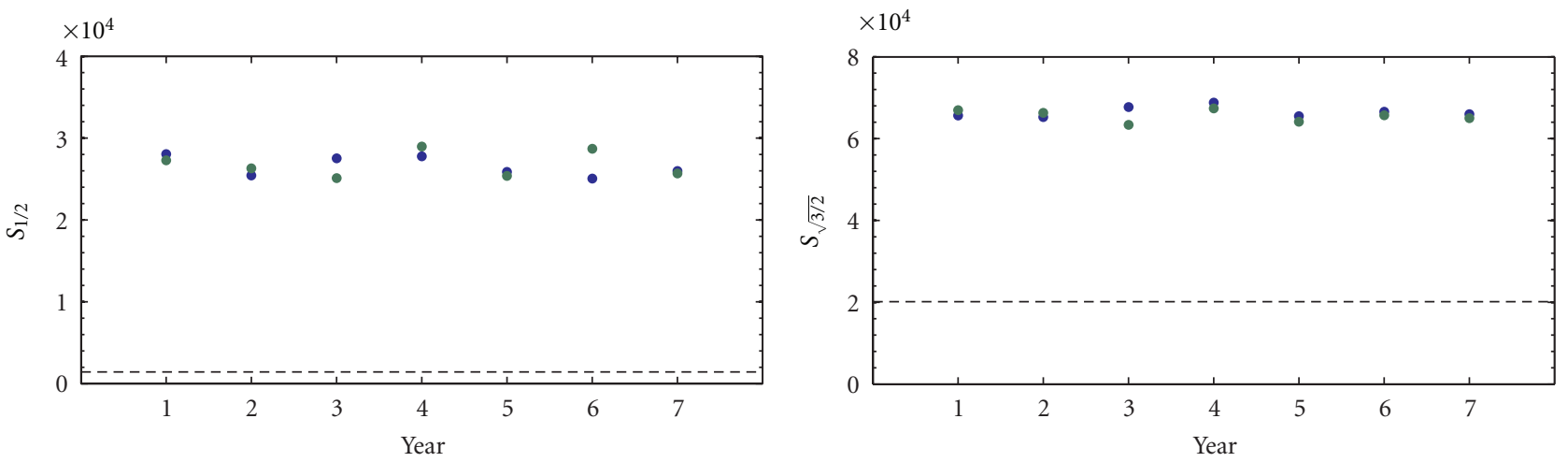

- V1

- V2

- V1

- V2

(a)

(b)
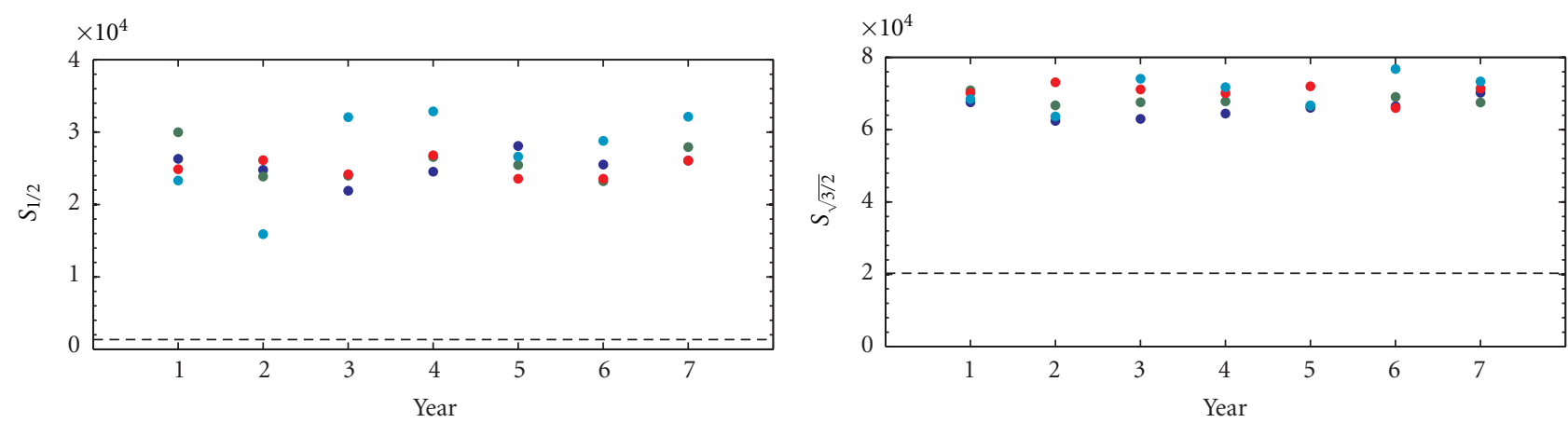

- W1 • W3

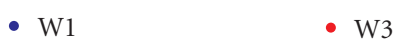

- W2

- W4

- W2

- W4

(c)

(d)

FIGURE 20: The $S$ statistics of the simulated data produced by the WMAP team. Dashed lines show the values of WMAP data.

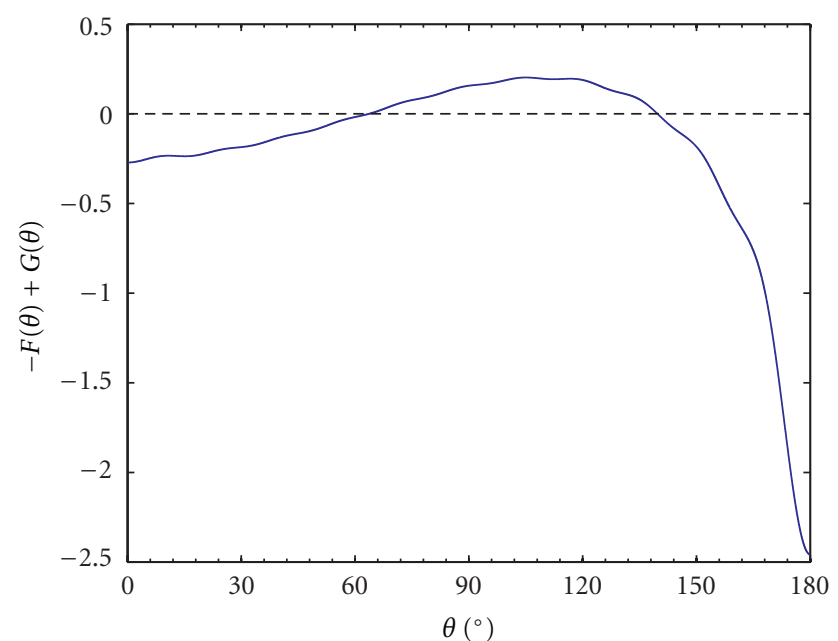

FIGURE 21: The effect of the odd multipole preference on the correlation.

consider the intervals $60^{\circ} \leq \theta \leq 120^{\circ}$ and $120^{\circ} \leq \theta \leq 180^{\circ}$, which are associated with the statistic $S_{1 / 2}$. At the interval

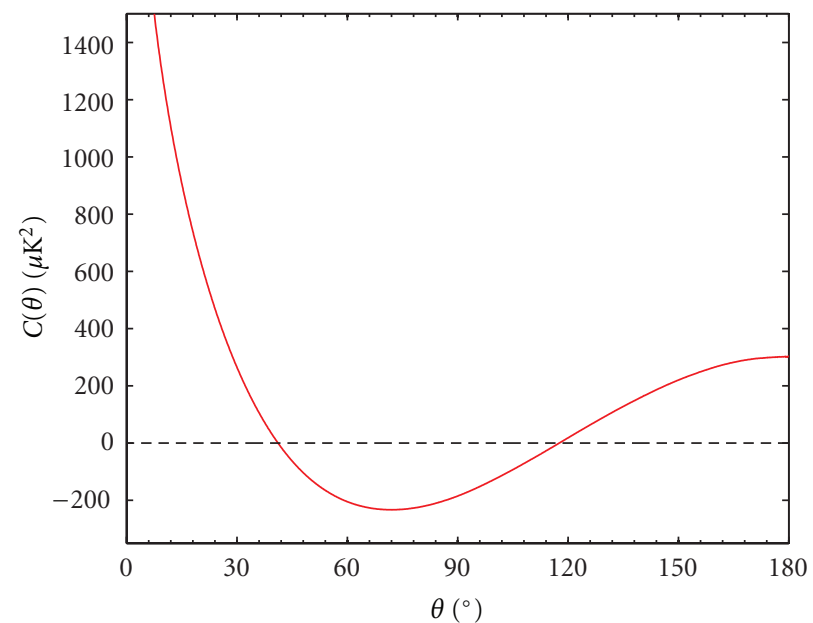

FIGURE 22: The angular correlation without odd-parity preference (i.e., (26)).

$60^{\circ} \leq \theta \leq 120^{\circ}$, the angular correlation has negative values, while the deviation $\alpha \varepsilon(-F(\theta)+G(\theta))$ is positive. At the 


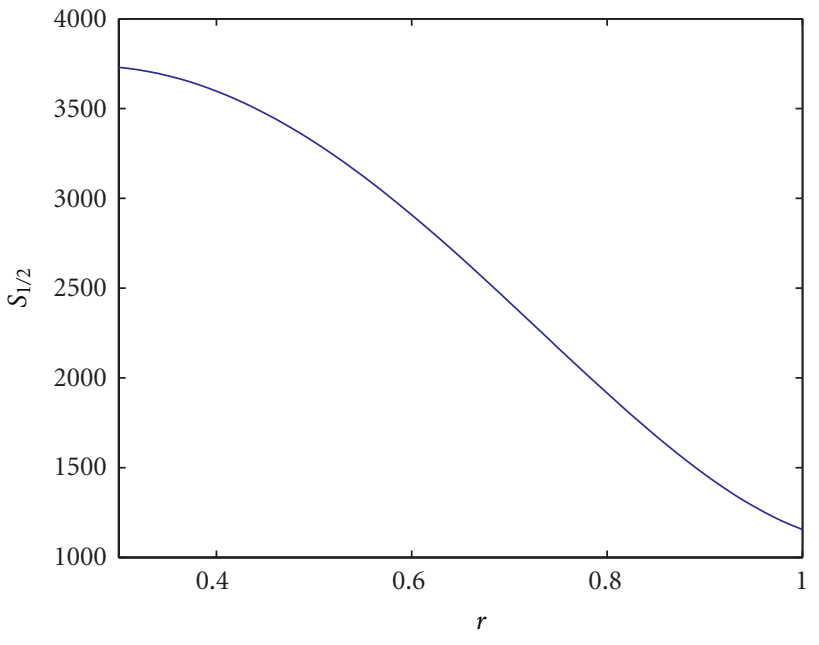

FIgURE 23: $S_{1 / 2}$ of the WMAP team's Internal Linear Combination (ILC) map, where the octupole components are multiplied by the suppression factor $r$.

interval $120^{\circ} \leq \theta \leq 180^{\circ}$, the angular correlation has positive values, while the deviation $\alpha \varepsilon(-F(\theta)+G(\theta))$ is negative. Therefore, we find

$$
\left(\left.C(\theta)\right|_{\varepsilon>0}\right)^{2}<\left(\left.C(\theta)\right|_{\varepsilon=0}\right)^{2} \quad\left(60^{\circ} \leq \theta \leq 180^{\circ}\right) .
$$

From (32), we may see that the odd-parity preference (i.e., $\epsilon>0$ ) leads to the lack of large-angle correlation power.

We like to stress that simple suppression of the power at a single multipole does not necessarily lead to the lack of largeangle correlation. For instance, suppressing octupole power, which mitigates the odd-parity preference, rather increases the large-angle correlation power. In Figure 23, we show $S_{1 / 2}$ of the WMAP team's Internal Linear Combination (ILC) map, where we have multiplied the suppression factor $r$ to the quadrupole component of the map. From Figure 23, we may see that the value of $S_{1 / 2}$ rather increases, as the octupole component is suppressed.

\section{Discussion}

We have investigated the symmetry and antisymmetry of the $\mathrm{CMB}$ anisotropy under the coordinate inversion, which are equivalent to the even and odd parity, respectively. As presented in this work, we find there is an anomalous oddparity preference at low multipole CMB data. We have investigated noncosmological origins and did not find definite association with known systematics. Among cosmological origins, topological models or primordial power spectrum of feature might provide theoretical explanation, though currently available models do not. One of a viable phenomenological model requires the real part of the primordial fluctuation to be suppressed at low wavenumbers, which leads to violation of translation invariance in primordial Universe on the scales larger than $4 \mathrm{Gpc}$. Additionally, we have compared the phase of even and odd multipole data and found they show behavior distinct from each other.
The WMAP power contrast anomaly between even and odd multipoles is explicitly associated with the angular power spectrum data, which are mainly used to fit a cosmological model. Having noted this, we have investigated whether even (odd) low multipole data set is consistent with the WMAP concordance model and found significant tension. We believe these parametric tensions indicate either unaccounted contamination or insufficiency of the assumed parametric model.

Noting the equivalence between the power spectrum and the correlation, we have investigated their association and found that the lack of large-angle correlation is phenomenologically identical with the odd-parity preference at low multipoles. Additionally, the low quadrupole power may be considered as a part of the odd-parity preference anomaly at low multipoles.

Depending on the type of cosmological origins, distinct anomalies are predicted in polarization data. Therefore, the upcoming Planck polarization data, which have low noise and large sky coverage, will greatly help us to understand the underlying origin of the anomaly.

\section{Acknowledgments}

The authors acknowledge the use of the Legacy Archive for Microwave Background Data Analysis (LAMBDA). Data analysis made the use of HEALPix [102, 103] and SpICE $[104,105]$. This work is supported in part by Danmarks Grundforskningsfond, which allowed the establishment of the Danish Discovery Center.

\section{References}

[1] P. Coles and F. Lucchin, Cosmology, Wiley, New York, NY, USA, 2nd edition, 2002.

[2] S. Dodelson, Modern Cosmology, Academic Press, New York, NY, USA, 2nd edition, 2003.

[3] V. Mukhanov, Physical Foundations of Cosmology, Cambridge University Press, New York, NY, USA, 1st edition, 2005.

[4] A. R. Liddle and D. H. Lyth, Cosmological Inflation and LargeScale Structure, Cambridge University Press, New York, NY, USA, 1st edition, 2000.

[5] S. Weinberg, Cosmology, Oxford University Press, New York, NY, USA, 1st edition, 2008.

[6] D. Larson, J. Dunkley, G. Hinshaw et al., "Seven-year Wilkinson Microwave Anisotropy Probe (WMAP) observations: power spectra and WMAP-derived parameters," The Astrophysical Journal, vol. 192, no. 2, article 16, 2011.

[7] N. Jarosik, C. L. Bennett, J. Dunkley et al., "Seven-year Wilkinson Microwave Anisotropy Probe (WMAP) observations: sky maps, systematic errors, and basic results," The Astrophysical Journal, vol. 192, no. 2, article 14, 2011.

[8] G. Hinshaw, J. L. Weiland, R. S. Hill et al., "Five-year Wilkinson Microwave Anisotropy Probe observations: data processing, sky maps and basic results," The Astrophysical Journal, vol. 180, no. 2, pp. 225-245, 2009.

[9] M. R. Nolta, J. Dunkley, R. S. Hill et al., "Five-year Wilkinson Microwave Anisotropy Probe observations: angular power spectra," The Astrophysical Journal, vol. 180, no. 2, pp. 296305, 2009. 
[10] J. Dunkley, E. Komatsu, M. R. Nolta et al., "Five-year Wilkinson Microwave Anisotropy Probe observations: likelihoods and parameters from the WMAP data," The Astrophysical Journal, vol. 180, no. 2, pp. 306-329, 2009.

[11] M. C. Runyan, P. A. R. Ade, R. S. Bhatia et al., "ACBAR: the arcminute cosmology bolometer array receiver," The Astrophysical Journal, vol. 149, no. 2, pp. 265-287, 2003.

[12] C. L. Reichardt, P. A. R. Ade, J. J. Bock et al., "High-resolution CMB power spectrum from the complete ACBAR data set," The Astrophysical Journal Letters, vol. 694, no. 2, pp. 1200 1219, 2009.

[13] P. Ade, J. Bock, M. Bowden et al., "First season QUaD $\mathrm{CMB}$ temperature and polarization power spectra," The Astrophysical Journal Letters, vol. 674, no. 1, pp. 22-28, 2008.

[14] C. Pryke, P. Ade, J. Bock et al., "Second and third season QUaD CMB temperature and polarization power spectra," The Astrophysical Journal, vol. 692, no. 2, Article ID 1247, 2009.

[15] J. Hinderks, P. Ade, J. Bock et al., "Parity violation constraints using cosmic microwave background polarization spectra from 2006 and 2007 observations by the QUaD polarimeter," Physical Review Letters, vol. 102, Article ID 161302, 4 pages, 2009.

[16] The Planck Collaboration, April 2006, The Scientific Programme of Planck, arXiv:astro-ph/0604069.

[17] J. C. Mather, D. J. Fixsen, R. A. Shafer, C. Mosier, and D. T. Wilkinson, "Calibrator design for the COBE Far Infrared Absolute Spectrophotometer (FIRAS)," The Astrophysical Journal Letters, vol. 512, no. 2, pp. 511-520, 1999.

[18] D. J. Fixsen, E. S. Cheng, J. M. Gales, J. C. Mather, R. A. Shafer, and E. L. Wright, "The cosmic microwave background spectrum from the full cobe firas data set," The Astrophysical Journal Letters, vol. 473, no. 2, pp. 576-587, 1996.

[19] C. L. Bennett, A. J. Banday, K. M. Górski et al., "Four-year cobe 1 DMR cosmic microwave background observations: maps and basic results," The Astrophysical Journal Letters, vol. 464, no. 1, pp. L1-L4, 1996.

[20] C. L. Bennett, R. S. Hill, G. Hinshaw et al., "Seven-year Wilkinson Microwave Anisotropy Probe (WMAP) observations: are there cosmic microwave background anomalies?" The Astrophysical Journal, vol. 192, no. 2, article 17, 2011.

[21] M. Cruz, E. Martínez-González, P. Vielva, and L. Cayón, "Detection of a non-gaussian spot in WMAP," Monthly Notices of the Royal Astronomical Society, vol. 356, no. 1, pp. 29-40, 2005.

[22] M. Cruz, M. Tucci, E. Martínez-González, and P. Vielva, "The non-gaussian cold spot in Wilkinson Microwave Anisotropy Probe: significance, morphology and foreground contribution," Monthly Notices of the Royal Astronomical Society, vol. 369, no. 1, pp. 57-67, 2006.

[23] M. Cruz, L. Cayón, E. Martínez-González, P. Vielva, and J. Jin, "The non-gaussian cold spot in the 3 year Wilkinson Microwave Anisotropy Probe data," The Astrophysical Journal Letters, vol. 655, no. 1 I, pp. 11-20, 2007.

[24] C. J. Copi, D. Huterer, and G. D. Starkman, "Multipole vectors: a new representation of the CMB sky and evidence for statistical anisotropy or non-gaussianity at $2 \leq \ell \leq 8$," Physical Review D, vol. 70, no. 4 A, Article ID 043515, p. 1, 2004.

[25] D. J. Schwarz, G. D. Starkman, D. Huterer, and C. J. Copi, "Is the low- $\ell$ microwave background cosmic?" Physical Review Letters, vol. 93, no. 22, Article ID 221301, 2004.

[26] C. J. Copi, D. Huterer, D. J. Schwarz, and G. D. Starkman, “On the large-angle anomalies of the microwave sky," Monthly
Notices of the Royal Astronomical Society, vol. 367, no. 1, pp. 79-102, 2006.

[27] K. Land and J. Magueijo, "Examination of evidence for a preferred axis in the cosmic radiation anisotropy," Physical Review Letters, vol. 95, no. 7, Article ID 071301, pp. 1-4, 2005.

[28] K. Land and J. Magueijo, "The axis of evil revisited," Monthly Notices of the Royal Astronomical Society, vol. 378, no. 1, pp. 153-158, 2007.

[29] A. Rakić and D. J. Schwarz, "Correlating anomalies of the microwave sky," Physical Review D, vol. 75, no. 10, Article ID 103002, 2007.

[30] C. J. Copi, D. Huterer, D. J. Schwarz, and G. D. Starkman, "Uncorrelated universe: statistical anisotropy and the vanishing angular correlation function in WMAP years 1-3," Physical Review D, vol. 75, no. 2, Article ID 023507, 2007.

[31] M. Hansen, A. M. Frejsel, J. Kim, P. Naselsky, and F. Nesti, "Pearson's random walk in the space of the CMB phases: evidence for parity asymmetry," Physical Review D, vol. 83, no. 10, Article ID 103508, 2011.

[32] P. D. Naselsky, L. Y. Chiang, P. Olesen, and O. V. Verkhodanov, "Primordial magnetic field and non-gaussianity of the one-year Wilkinson Microwave Anisotropy Probe data," The Astrophysical Journal Letters, vol. 615, no. 1 I, pp. 45-54, 2004.

[33] L. Y. Chiang, P. D. Naselsky, O. V. Verkhodanov, and M. J. Way, "Non-gaussianity of the derived maps from the first-year Wilkinson Microwave Anisotropy Probe data," The Astrophysical Journal Letters, vol. 590, no. 2, pp. L65-L68, 2003.

[34] J. Kim and P. Naselsky, "Cosmological Alfvén waves in the recent $\mathrm{CMB}$ data, and the observational bound on the primordial vector perturbation," Journal of Cosmology and Astroparticle Physics, vol. 7, article 041, 2009.

[35] P. Naselsky, L. Y. Chiang, P. Olesen, and I. Novikov, "Statistics of phase correlations as a test for non-gaussianity of the CMB maps," Physical Review D, vol. 72, no. 6, Article ID 063512, 12 pages, 2005.

[36] P. Coles, P. Dineen, J. Earl, and D. Wright, "Phase correlations in cosmic microwave background temperature maps," Monthly Notices of the Royal Astronomical Society, vol. 350, no. 3, pp. 989-1004, 2004.

[37] J. Kim and P. Naselsky, "Anomalous parity asymmetry of the Wilkinson Microwave Anisotropy Probe power spectrum data at low multipoles," The Astrophysical Journal Letters, vol. 714, no. 2, pp. L265-L267, 2010.

[38] J. Kim and P. Naselsky, "Anomalous parity asymmetry of WMAP 7-year power spectrum data at low multipoles: is it cosmological or systematics?" Physical Review D, vol. 82, no. 6, Article ID 063002, 2010.

[39] A. Gruppuso, F. Finelli, P. Natoli et al., "New constraints on parity symmetry from a re-analysis of the WMAP-7 low-resolution power spectra," Monthly Notices of the Royal Astronomical Society, vol. 411, no. 3, pp. 1445-1452, 2011.

[40] J. Kim and P. Naselsky, "Parametric tension between even and odd multipole data of the WMAP power spectrum: unaccounted contamination or missing parameters?" The Astrophysical Journal Letters, vol. 724, no. 2, pp. L217-L220, 2010.

[41] J. Kim and P. Naselsky, "Lack of angular correlation and oddparity preference in cosmic microwave background data," The Astrophysical Journal, vol. 739, no. 2, Article ID 79, 2011.

[42] H. K. Eriksen, F. K. Hansen, A. J. Banday, K. M. Górski, and P. B. Lilje, "Erratum: asymmetries in the cosmic microwave background anisotropy field," The Astrophysical Journal, vol. 609, no. 2 I, pp. 1198-1199, 2004. 
[43] J. Hoftuft, H. K. Eriksen, A. J. Banday, K. M. Górski, F. K. Hansen, and P. B. Lilje, "Increasing evidence for hemispherical power asymmetry in the five-year wmap data," The Astrophysical Journal Letters, vol. 699, no. 2, pp. 985-989, 2009.

[44] A. De Oliveira-Costa, M. Tegmark, M. Zaldarriaga, and A. Hamilton, "Significance of the largest scale CMB fluctuations in WMAP," Physical Review D, vol. 69, no. 6, Article ID 063516, 2004.

[45] K. Land and J. Magueijo, "Is the Universe odd?" Physical Review D, vol. 72, no. 10, Article ID 101302, 5 pages, 2005.

[46] G. Hinshaw, A. J. Banday, C. L. Bennett et al., "Twopoint correlations in the COBE 1 DMR four-year anisotropy maps," The Astrophysical Journal Letters, vol. 464, no. 1, pp. L25-L28, 1996.

[47] D. N. Spergel, L. Verde, H. V. Peiris et al., "First-year Wilkinson Microwave Anisotropy Probe (WMAP) observations: determination of cosmological parameters," The Astrophysical Journal, vol. 148, no. 1, pp. 175-194, 2003.

[48] C. J. Copi, D. Huterer, D. J. Schwarz, and G. D. Starkman, "No large-angle correlations on the non-galactic microwave sky," Monthly Notices of the Royal Astronomical Society, vol. 399, no. 1, pp. 295-303, 2009.

[49] D. Huterer, C. J. Copi, D. J. Schwarz, and G. D. Starkman, "Large-angle anomalies in the CMB," Advances in Astronomy, vol. 2010, Article ID 847541, 2010.

[50] C. J. Copi, D. Huterer, D. J. Schwarz, and G. D. Starkman, "Bias in low-multipole CMB reconstructions," March 2011, arXiv:1103.3505.

[51] G. B. Arfken and H. J. Weber, Mathematical Methods for Physicists, Academic Press, San Diego, Calif, USA, 5th edition, 2000.

[52] G. Hinshaw et al., "Three-year Wilkinson Microwave Anisotropy Probe (WMAP) observations: temperature analysis," The Astrophysical Journal, vol. 170, article 288, 2007.

[53] E. Komatsu, J. Dunkley, M. R. Nolta et al., "Five-year Wilkinson Microwave Anisotropy Probe observations: cosmological interpretation," The Astrophysical Journal, vol. 180, no. 2, pp. 330-376, 2009.

[54] E. Komatsu, K. M. Smith, J. Dunkley et al., "Seven-year Wilkinson Microwave Anisotropy Probe (WMAP) observations: cosmological interpretation," The Astrophysical Journal, vol. 192, no. 2, article 18, 2011.

[55] J. R. Bond, A. H. Jaffe, and L. Knox, "Estimating the power spectrum of the cosmic microwave background," Physical Review D, vol. 57, no. 4, pp. 2117-2137, 1998.

[56] G. Efstathiou, "Hybrid estimation of cosmic microwave background polarization power spectra," Monthly Notices of the Royal Astronomical Society, vol. 370, no. 1, pp. 343-362, 2006.

[57] B. D. Wandelt, E. Hivon, and K. M. Górski, "Cosmic microwave background anisotropy power spectrum statistics for high precision cosmology," Physical Review D, vol. 64, no. 8, Article ID 083003, 2001.

[58] E. Hivon, K. M. Górski, C. B. Netterfield, B. P. Crill, S. Prunet, and F. Hansen, "Master of the cosmic microwave background anisotropy power spectrum: a fast method for statistical analysis of large and complex cosmic microwave background data sets," The Astrophysical Journal Letters, vol. 567, no. 1 I, pp. 2-17, 2002.

[59] R. S. Hill, J. L. Weiland, N. Odegard et al., "Five-year Wilkinson Microwave Anisotropy Probe observations: beam maps and window functions," The Astrophysical Journal, vol. 180, no. 2, pp. 246-264, 2009.
[60] B. Gold, N. Odegard, J. L. Weiland et al., "Seven-year Wilkinson Microwave Anisotropy Probe (WMAP) observations: galactic foreground emission," The Astrophysical Journal, vol. 192, no. 2, article 15, 2011.

[61] D. P. Finkbeiner, M. Davis, and D. J. Schlegel, "Extrapolation of galactic dust emission at 100 microns to cosmic microwave background radiation frequencies using FIRAS," The Astrophysical Journal Letters, vol. 524, no. 2, pp. 867-886, 1999.

[62] D. P. Finkbeiner, "A full-sky $\mathrm{H} \alpha$ template for microwave foreground prediction," The Astrophysical Journal, vol. 146, no. 2, pp. 407-415, 2003.

[63] C. L. Bennett, R. S. Hill, G. Hinshaw et al., "First-year Wilkinson Microwave Anisotropy Probe (WMAP) observations: foreground emission," The Astrophysical Journal, vol. 148, no. 1, pp. 97-117, 2003

[64] M. Tegmark and G. Efstathiou, "A method for subtracting foregrounds from multifrequency CMB sky maps," Monthly Notices of the Royal Astronomical Society, vol. 281, no. 4, pp. 1297-1314, 1996.

[65] B. Gold, C. L. Bennett, R. S. Hill et al., "Five-year Wilkinson Microwave Anisotropy Probe observations: galactic foreground emission," The Astrophysical Journal, vol. 180, no. 2, pp. 265-282, 2009.

[66] G. Rieke, Detection of Light: From the Ultraviolet to Submillimeter, Cambridge University Press, New York, NY, USA, 2nd edition, 2002.

[67] G. Hinshaw, C. Barnes, C. L. Bennett et al., "First-year Wilkinson Microwave Anisotropy Probe (WMAP) observations: data processing methods and systematic error limits," The Astrophysical Journal, vol. 148, no. 1, pp. 63-95, 2003.

[68] N. Jarosik, C. Barnes, M. R. Greason et al., "Three-year Wilkinson Microwave Anisotropyprobe (WMAP)1 observations: beam profiles, data processing, radiometer characterization, and systematic error limits," The Astrophysical Journal, vol. 170, no. 2, pp. 263-287, 2007.

[69] I. K. Wehus, L. Ackerman, H. K. Eriksen, and N. E. Groeneboom, "The effect of asymmetric beams in the Wilkinson Microwave Anisotropy Probe experiment," The Astrophysical Journal Letters, vol. 707, no. 1, pp. 343-353, 2009.

[70] G. Efstathiou, "The statistical significance of the low cosmic microwave background mulitipoles," Monthly Notices of the Royal Astronomical Society, vol. 346, no. 2, pp. L26-L30, 2003.

[71] A. Niarchou and A. Jaffe, "Imprints of spherical nontrivial topologies on the cosmic microwave background," Physical Review Letters, vol. 99, no. 8, Article ID 081302, 2007.

[72] K. Land and J. Magueijo, "Template fitting and the largeangle cosmic microwave background anomalies," Monthly Notices of the Royal Astronomical Society, vol. 367, no. 4, pp. 1714-1720, 2006.

[73] J. Martin and R. H. Brandenberger, "Trans-planckian problem of inflationary cosmology," Physical Review D, vol. 63, no. 12, Article ID 123501, 2001.

[74] J. Martin and R. Brandenberger, "Dependence of the spectra of fluctuations in inflationary cosmology on trans-Planckian physics," Physical Review D, vol. 68, no. 6, Article ID 063513, 2003.

[75] U. H. Danielsson, "Note on inflation and trans-Planckian physics," Physical Review D, vol. 66, no. 2, Article ID 023511, 2002.

[76] R. Easther, B. R. Greene, W. H. Kinney, and G. Shiu, "Generic estimate of trans-Planckian modifications to the primordial power spectrum in inflation," Physical Review D, vol. 66, no. 2, Article ID 023518, 2002. 
[77] N. Kaloper, M. Kleban, A. Lawrence, and S. Shenker, "Signatures of short distance physics in the cosmic microwave background," Physical Review D, vol. 66, no. 12, Article ID 123510, 2002.

[78] J. Martin and C. Ringeval, "Superimposed oscillations in the WMAP data?" Physical Review D, vol. 69, no. 8, Article ID 083515, 2004.

[79] C. P. Burgess, J. M. Cline, F. Lemieux, and R. Holman, "Are inflationary predictions sensitive to very high energy physics?" Journal of High Energy Physics, vol. 2, article 048, 2003.

[80] K. Schalm, G. Shiu, and J. P. Van Der Schaar, "Decoupling in an expanding universe: boundary RG-flow affects initial conditions for inflation," Journal of High Energy Physics, vol. 4, article 076, 2004.

[81] R. Easther, W. H. Kinney, and H. Peiris, "Boundary effective field theory and trans-Planckian perturbations: astrophysical implications," Journal of Cosmology and Astroparticle Physics, vol. 8, article 001, 2005.

[82] R. Easther, W. H. Kinney, and H. Peiris, "Observing transPlanckian signatures in the cosmic microwave background," Journal of Cosmology and Astroparticle Physics, vol. 5, article 009, 2005.

[83] S. L. Bridle, A. M. Lewis, J. Weller, and G. Efstathiou, "Reconstructing the primordial power spectrum," Monthly Notices of the Royal Astronomical Society, vol. 342, no. 4, pp. L72-L78, 2003.

[84] G. Nicholson, C. R. Contaldi, and P. Paykari, "Reconstruction of the primordial power spectrum by direct inversion," Journal of Cosmology and Astroparticle Physics, vol. 1, article 016, 2010.

[85] J. Hamann, A. Shafieloo, and T. Souradeep, "Features in the primordial power spectrum? A frequentist analysis," Journal of Cosmology and Astroparticle Physics, vol. 4, article 010, 2010.

[86] S. Alexander, T. Biswas, A. Notari, and D. Vaid, "Local void vs dark energy: confrontation with WMAP and type Ia supernovae," Journal of Cosmology and Astroparticle Physics, vol. 9, article 025, 2009.

[87] T. Clifton, P. G. Ferreira, and K. Land, "Living in a void: testing the copernican principle with distant supernovae," Physical Review Letters, vol. 101, no. 13, Article ID 131302, 2008.

[88] J. M. Bardeen, "Gauge-invariant cosmological perturbations," Physical Review D, vol. 22, no. 8, pp. 1882-1905, 1980.

[89] N. I. Fisher, Statistical analysis of Circular Data, Cambridge University Press, New York, NY, USA, 1st edition, 1993.

[90] P. G. Ferreira, J. Magueijo, and K. M. Górski, "Evidence for non-gaussianity in the cobe DMR 4 year sky maps," The Astrophysical Journal Letters, vol. 503, no. 1, pp. L1-L4, 1998.

[91] K. V. Mardia and P. E. Jupp, Directional Statistics, Wiley, New York, NY, USA, 1st edition, 1999.

[92] A. Lewis and S. Bridle, "Cosmological parameters from CMB and other data: a Monte Carlo approach," Physical Review D, vol. 66, no. 10, Article ID 103511, 2002.

[93] H. K. Eriksen, I. J. O’Dwyer, J. B. Jewell et al., "Power spectrum estimation from high-resolution maps by Gibbs sampling," The Astrophysical Journal, vol. 155, no. 2, pp. 227 241, 2004.

[94] A. Lewis and S. Bridle, Cosmomc++. 8, 2006.

[95] A. Gelman and D. B. Rubin, "Inference from iterative simulation using multiple sequences," Statistical Science, vol. 7, article 457, 1992.
[96] S. P. Brooks and A. Gelman, "General methods for monitoring convergence of iterative simulations," Journal of Computational and Graphical Statistics, vol. 7, no. 4, pp. 434-455, 1998.

[97] A. M. Mood, Introduction to the Theory of Statistics, McGrawHill, New York, NY, USA, 3rd edition, 1974.

[98] D. R. Cox and D.V. Hinkley, Theoretical Statistics, Chapman and Hall, London, UK, 1st edition, 1979.

[99] R. Lupton, Statistics in Theory and Practice, Princeton University Press, Princeton, NJ, USA, 1st edition, 1993.

[100] S. J. Bence, K. F. Riley, and M. P. Hobson, Mathematical Methods for Physics and Engineering: a Comprehensive Guide, Cambridge University Press, New York, NY, USA, 3rd edition, 2006.

[101] T. Padmanabhan, Structure Formation in the Universe, Cambridge University Press, New York, NY, USA, 1st edition, 1993.

[102] K. M. Gorski, B. D. Wandelt, F. K. Hansen, E. Hivon, and A. J. Banday, “The HEALPix primer," May 1999, arXiv:astroph/9905275.

[103] M. Górski, E. Hivon, A. J. Banday et al., "HEALPix: a framework for high-resolution discretization and fast analysis of data distributed on the sphere," The Astrophysical Journal, vol. 622, no. 2 I, pp. 759-771, 2005.

[104] I. Szapudi, S. Prunet, D. Pogosyan, A. S. Szalay, and J. R. Bond, "Fast cosmic microwave background analyses via correlation functions," The Astrophysical Journal Letters, vol. 548, no. 2, pp. L115-L118, 2001.

[105] I. Szapudi, S. Prunet, and S. Colombi, "Fast analysis of inhomogenous megapixel cosmic microwave background maps," The Astrophysical Journal Letters, vol. 561, no. 1, pp. L11-L14, 2001. 

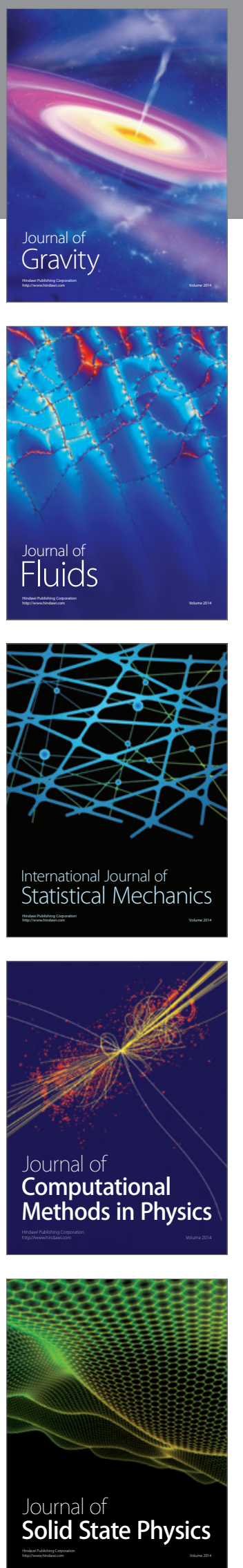

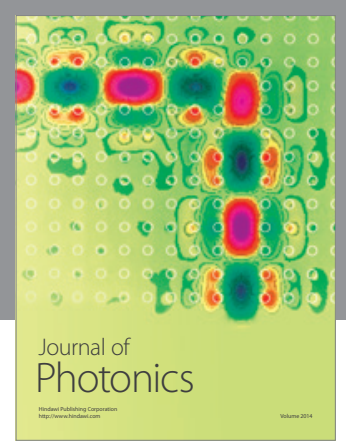

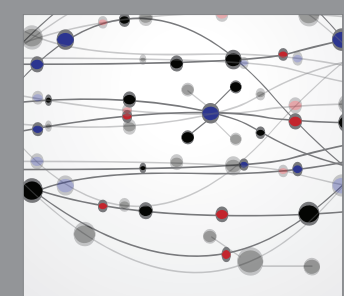

The Scientific World Journal
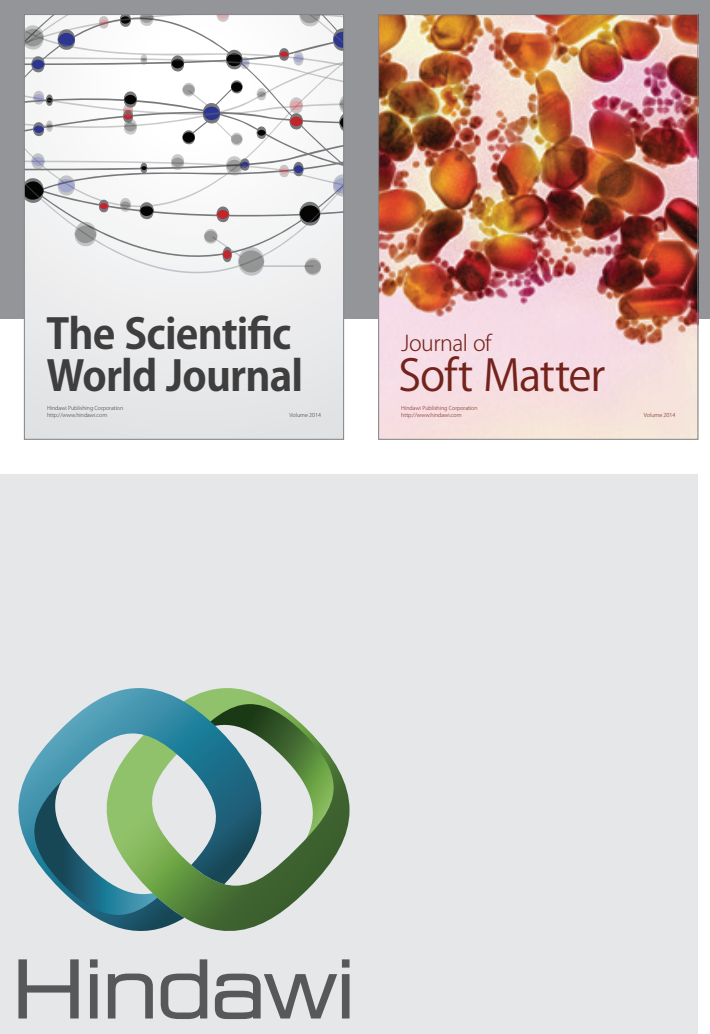

Submit your manuscripts at

http://www.hindawi.com
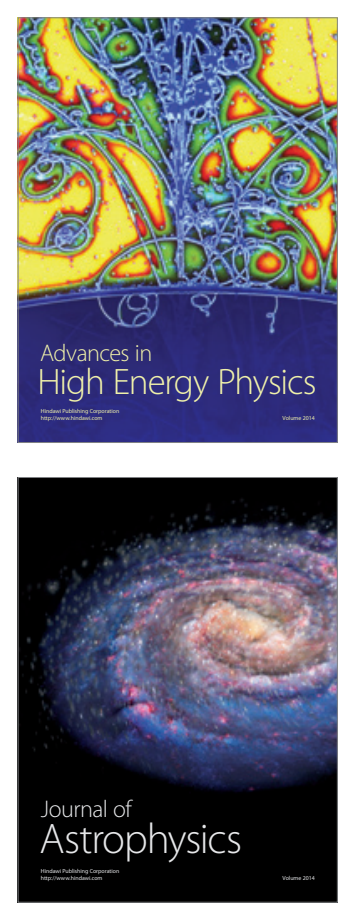
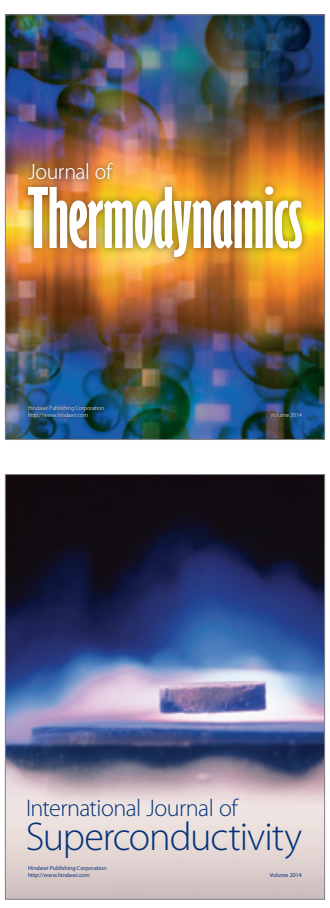
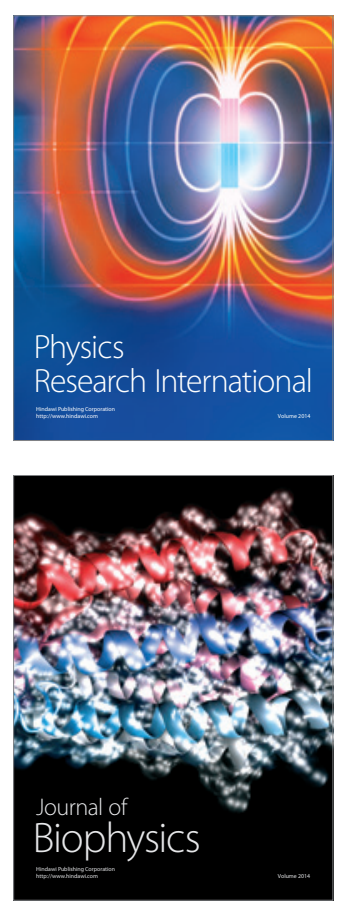
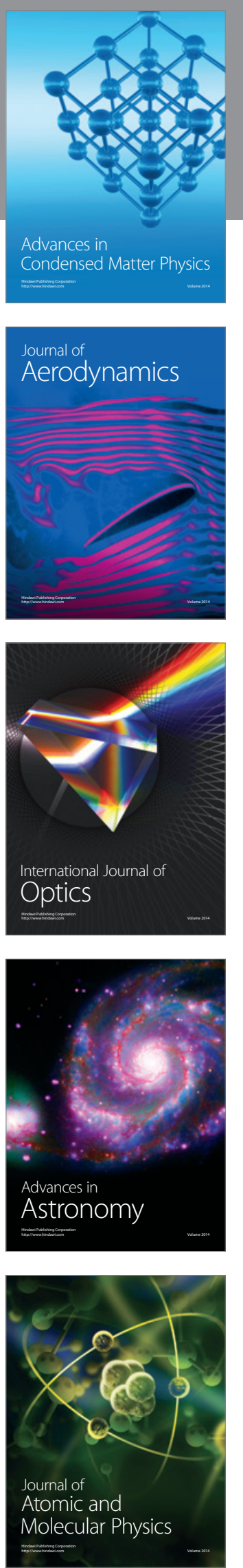